Pacific Journal of Mathematic 


\title{
TOTAL POSITIVITY OF CERTAIN REPRODUCING KERNELS
}

\author{
JACOB BURBEA
}

In this paper we study the total positivity of various kernels, especially reproducing kernels of Hilbert spaces of analytic functions. We do so by employing a familiar device known as the "composition formula of Pólya and Szegö." Using this formula we are able to give a short proof of the variation diminishing property of a generalized analogue of the la Vallee Poussin means. This generalizes earlier work of Pólya and Schoenberg and recent work of Horton. Our method is also based on the isometrical image of the reproducing kernel called the generating function. The reproducing kernel is then expressed as a composition of two generating functions so that the problem is reduced to investigating the total positivity of the generating function. This methods extends earlier work and yields many new reproducing kernels which are total positive.

1. Introduction. The theory of total positivity and more generally the theory of sign regularity have been extensively applied in various fields of mathematics and in particular in the theory of approximation [9, 10]. In a previous paper [5] it was shown that the optimality of a quadrature formula is closely connected with the notion of the total positivity of the reducing kernel of the functions determining the formula (cf. Karlin [10]). In [5] the notion of total positivity was extended in a natural manner to domains in the complex plane. For simply connected domains, for which the reproducing kernel is an automorphic form of arbitrary weight, it was shown that the reproducing kernel is indeed totally positive thereby yielding a differential geometric interpretation of total positivity. It was also shown that in general, reproducing kernels of multiply connected domains are not totally positive. The methods in [5] however cannot be applied to reproducing kernels which are not automorphic forms and the purpose of this paper is to establish the total positivity of such kernels. We do this by employing a familiar device (Karlin [9, p. 98]) known as the "composition formula of Pólya and Szegö." As mentioned by Karlin, this is the only device known to us as a binary operation, that permits us to construct a totally positive kernel from two such kernels.

Using the above composition formula and an explicit formula for Jacobi polynomials due to Bateman [3], we will give a very short proof of a theorem, proved first by Horton [8], on the variation 
diminishing property of an analogue of de la Vallée Poussin means. This generalizes earlier work of Pólya and Schoenberg [12] and the work of Horton [8].

Our method is also based on the isometrical image of the reproducing kernel called the generating function (cf. [15]). The reproducing kernel is then expressed as a composition of two generating functions. Hence, the problem is reduced to investigating the total positivity of the generating function. The method yields many new reproducing kernels which are totally positive. Explicitly, kernels which are derived from the generalized hypergeometric functions are in general totally positive. This extends our previous work [5].

2. Total positivity. We introduce some notation and definitions from the theory of total positivity. We shall use Karlin's book [9] (especially pp. 1-103) as a basic reference (see also [5]).

A real function (or kernel) $K(x, y)$ of two variables ranging over linearly ordered sets $A$ and $B$, respectively, is said to be sign regular (abbreviated SR) on $A \times B$ if for all $m=1,2, \cdots, x_{1}<x_{2}<\cdots<x_{m}$, $y_{1}<y_{2}<\cdots<y_{m}, x_{i} \in A, y_{i} \in B$, we have the inequalities

$$
\varepsilon_{m} K\left(\begin{array}{l}
x_{1}, \cdots, x_{m} \\
y_{1}, \cdots, y_{m}
\end{array}\right)=\varepsilon_{m} \operatorname{det}\left\|K\left(x_{i}, y_{j}\right)\right\|_{i, j=1}^{m} \geqq 0
$$

for a sequence of signs $\varepsilon_{m}= \pm 1$. $\varepsilon_{m}=\varepsilon_{m}(K)$ is also called the $m$ th order signature of $K, K \equiv K(x, y)$. When $\varepsilon_{m}=1, m=1,2, \cdots$, we say that $K$ is totally positive (TP). If $\varepsilon_{m}=(-1)^{m(m-1) / 2}, m=1,2, \cdots, K$ is said to be sign-reverse regular (RR). If in (2.1) strict inequality holds for $m=1,2, \cdots, K$ is said to be strictly SR (SSR), strictly TP (STP) or strictly RR (SRR) respectively.

Let $K(x, y)$ be of class $\mathcal{C}^{\infty}(A \times B) . \quad K(x, y)$ is said to be extended SR (ESR) on $A \times B$ if for $m=1,2, \cdots$, we have the inequalities

$$
\varepsilon_{m} K^{*}(\overbrace{\left.\begin{array}{l}
x, \cdots, x \\
y, \cdots, y
\end{array}\right)}^{m}=\varepsilon_{m} \operatorname{det}\left\|\frac{\partial^{k+j-2}}{\partial x^{k-1} \partial y^{j-1}} K(x, y)\right\|_{k, j=1}^{m}>0
$$

for a sequence of signs (signatures) $\varepsilon_{m}= \pm 1$. If $\varepsilon_{m}=+1, m=1,2, \cdots$, we say that $K$ is extended TP (ETP). If $\varepsilon_{m}=(-1)^{m(m-1) / 2}, m=1,2, \cdots$, $K$ is said to be extended RR (ERR).

If $K(x, y)$ is ESR (ETP or ERR) on $A \times B$ then [9, p. 55] $K(x, y)$ is SSR (STP or SRR) on $A \times B$.

The notion of ESR can be extended to the complex plane as follows: Let $D$ be a domain in the complex plane. $K(z, \bar{\zeta})$ of class $\widetilde{c}^{\infty}$ in the two complex variables $(z, \bar{\zeta}),(z, \zeta) \in D \times D$, is said to be 
ESR on $D$ if, for $m=1,2, \cdots$, we have the inequalities

$$
K^{*}(\overbrace{z, \cdots, z}^{m} \begin{array}{l}
z, \cdots, \bar{\zeta}
\end{array})=\operatorname{det}\left\|\frac{\partial^{k+j-2}}{\partial z^{k-1} \partial \bar{\zeta}^{j-1}} K(z, \bar{\zeta})\right\|_{k, j=1}^{m} \neq 0 .
$$

Let $\gamma$ be an arc in $D . K(z, \bar{\zeta})$ is said to be $\operatorname{ESR}$ on $\gamma$ if

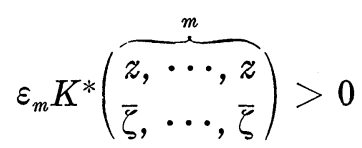

for all $(z, \bar{\zeta}),(z, \zeta) \in \gamma \times \gamma$, and for $\varepsilon_{m}= \pm 1$. If $\varepsilon_{m}=+1, m=1,2, \cdots$, $K(z, \bar{\zeta})$ is said to be ETP on $\gamma$. Similarly, if $\varepsilon_{m}=(-1)^{m(m-1) / 2}$, $m=1,2, \cdots, K(z, \bar{\zeta})$ is said to be ERR on $\gamma$.

Analogously, we can introduce the notion of ESR, respectively of ETP and ERR, for a kernel $K(z, \bar{\zeta})$ defined on $A_{1} \times A_{2}$, where $A_{1}$ and $A_{2}$ are sets in the complex plane.

The "basic composition formula" [9, pp. 16-17] below plays an essential role in establishing the total positivity of the kernels considered in our work. Suppose $\sigma(t)$ is a sigma-finite measure defined on $A, A \subset \boldsymbol{R}$. Let $K, L$ and $M$ be Borel measurable functions of two variables satisfying

$$
K(z, \bar{\zeta})=\int_{A} L(t, z) M(t, \bar{\zeta}) d \sigma(t), \quad(z, \zeta) \in D \times D,
$$

where the integral is assumed to converge absolutely. We have (cf. [9, p. 99]):

Proposition 1. Let the assumptions of formula (2.2) prevail, and let $\gamma$ be an arc in $D$. Then

(a) If $L(t, z)$ and $M(t, \bar{\zeta})$ are SR (SSR or ESR) on $A \times \gamma$ and $A \times \tilde{\gamma}(\tilde{\gamma}=\{\bar{\zeta}: \zeta \in \gamma\})$ respectively then $K(z, \bar{\zeta})$ is SR (SSR or ESR) on $\gamma$.

(b) If $L(t, z)$ and $M(t, \bar{\zeta})$ are SR (SSR or ESR) and of the same signatures on $A \times \gamma$ and $A \times \widetilde{\gamma}$ respectively then $K(z, \bar{\zeta})$ is TP (STP or ETP) on $\gamma$.

For ready reference we also record in the form of a proposition two simple properties pertinent to SR kernels (cf. [9, p. 18]). We formulate the proposition in only the case of ESR. The cases of SR and SSR hold true after minor modifications.

Proposition 2. (a ) If $K(z, \bar{\zeta})$ is ESR on $A_{1} \times A_{2}$ and $\varphi(z)$ and $\psi(\zeta)$ are nonvanishing ${ }^{\infty}$-functions on $A_{1}$ and $A_{2}$ respectively, then $L(z, \bar{\zeta})=\varphi(z) \overline{\psi(\zeta)} K(z, \bar{\zeta})$ is ESR on $A_{1} \times A_{2}$. In fact 


$$
L^{*}(\overbrace{\left.\begin{array}{l}
z, \cdots, z \\
\bar{\zeta}, \cdots, \bar{\zeta}
\end{array}\right)}^{m}=[\varphi(z) \overline{\psi(\zeta)}]^{m} K^{*}(\overbrace{\left.\begin{array}{l}
z, \cdots, z \\
\bar{\zeta}, \cdots, \bar{\zeta}
\end{array}\right)}^{m} .
$$

(b) If $K(z, \bar{\zeta})$ is ESR on $A_{1} \times A_{2}$ and $w=\varphi(z)$ and $\tau=\psi(\zeta)$ are schlicht on $A_{1}$ and $A_{2}$ respectively, then

$$
L(w, \bar{\tau})=K\left(\Phi^{-1}(w), \overline{\psi^{-1}(\tau)}\right)
$$

is $\operatorname{ESR}$ on $\varphi\left(A_{1}\right) \times \psi\left(A_{2}\right)$. In fact

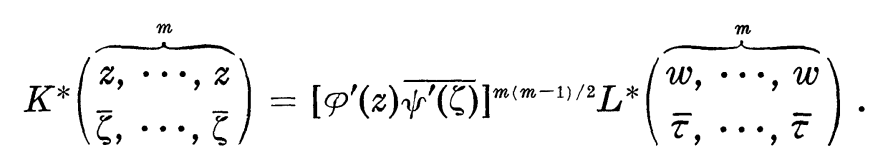

The following proposition is mentioned in [9, p. 101].

Proposition 3. Let $K_{N}(x, y)=\sum_{n=0}^{N} a_{n}[u(x)]^{n}[v(y)]^{n}, a_{n}>0, n=$ $0,1, \cdots, N$, and $u(x)$ and $v(y)$ are positive functions on the real intervals $I$ and $J$ respectively. Then

(a) If $u$ and $v$ are increasing (decreasing) on $I$ and $J$ respectively, then $K_{N}(x, y)$ is $\mathrm{TP}$ on $I \times J$.

(b) If $u$ is increasing (decreasing) and $v$ is decreasing (increasing) on $I$ and $J$ respectively, then $K_{N}(x, y)$ is $R R$ on $I \times J$.

(c) The above statements true for $N=\infty$ provided the bilinear sum converges. In that case, if in addition $u$ and $v$ are $\mathbb{C}^{\infty}$-functions, then $K_{\infty}(x, y)$ is ETP on $I \times J$ in case (a) and ERR on $I \times J$ in case (b).

Proof. All these follow from

$$
K_{N}(x, y)=\int_{R} e^{t \operatorname{lc} g u(x)} e^{t \log v(y)} d \mu_{N}(t)
$$

where $\mu_{N}$ is a discrete measure with jumps $a_{n}$ at $n=0,1, \cdots, N$, and the fact that $e^{x y}$ is ETP, $e^{-x y}$ is ERR, and Proposition 2.

CoROLlaRY 1. (a) $K_{n}(x, y)=(1-x)^{n}(1-y)^{n} \sum_{k=0}^{n} a_{k c}((1+x) /(1-x))^{k}$ $((1+y) /(1-y))^{k}, a_{k}>0, k=0,1, \cdots, n$, is TP on $(-1,1) \times(-1,1)$.

(b) $L_{n}(x, y)=K_{n}(-x, y)$ is RR on $(-1,1) \times(-1,1)$.

Proof. Indeed, $1-x>0, v(x)=(1+x) /(1-x)>0$ and $v^{\prime}(x)>0$ on $(-1,1)$.

3. Variation diminishing property-Jacobi polynomials. Let $f(t)$ be defined on an ordered set of the real line $J$. Write 


$$
V_{J}[f]=\operatorname{Sup} V\left[f\left(t_{1}\right), f\left(t_{2}\right), \cdots, f\left(t_{n}\right)\right]
$$

where the supremum is extended over all sets $t_{1}<t_{2}<\cdots<t_{n}$, $t_{i} \in J$ and $V\left(\alpha_{1}, \alpha_{2}, \cdots, \alpha_{n}\right)$ is the number of sign changes of the real sequence $\alpha_{1}, \alpha_{2}, \cdots, \alpha_{n}$, zero terms being discarded. A real-valued kernel $K(x, y)$ defined on $I \times J$ is said to be variation diminishing (VD) on $I$ if,

$$
V_{I}[K * f] \leqq V_{J}[f] \text { for all } f \in L_{1}(\mu, J) \text {. }
$$

Here $\mu$ is a positive measure on $J$ and the convolution

$$
(K * f)(x)=\int_{J} K(x, y) f(y) d \mu(y)
$$

is assumed to be finite for every $x$ in the ordered set of the real line $I$.

If $g$ is a $\widetilde{c}^{\infty}$-function on an open set $I, Z_{I}[g]$ will denote the number of zeros, counting multiplicity, of $g$ in $I$. We write $\bar{A}$ for the closure of $A$ in $\boldsymbol{R}$.

The following proposition is a much weaker statement (but it is all that we need here) than the one found in Karlin [9, p. 21].

Proposition 4. Let $I$ and $J$ be open in $R$. Assume that $K(x, y)$ is in $\widetilde{C}^{\infty}(\bar{I} \times \bar{J})$ and SR on $I \times J$. Then $K(x, y)$ is VD on $I$ and in fact

$$
V_{\bar{I}}[K * f] \leqq Z_{I}[K * f] \leqq V_{\bar{J}}[f]
$$

for all $f \in L_{1}(\mu, J)$.

The first inequality is obvious due to the continuity of $K * f$ in $I$ while the second inequality is the content of Karlin's theorem.

CoRollary 2. Let $K_{n}(x, y)$ and $L_{n}(x, y)$ be defined as in (a) and (b) of Corollary 1. Then

$$
V_{[-1,1]}\left[K_{n} * f\right] \leqq Z_{(-1,1)}\left[K_{n} * f\right] \leqq V_{[-1,1]}[f]
$$

and

$$
V_{[-1,1]}\left[L_{n} * f\right] \leqq Z_{(-1,1)}\left[L_{n} * f\right] \leqq V_{[-1,1]}[f],
$$

for all $f \in L_{1}(\mu)$, where $\mu(x)=(1-x)^{\alpha}(1+x)^{\beta}$ on $[-1,1], \alpha, \beta>-1$.

The Jacobi polynomials, $P_{n}^{(\alpha, \beta)}(x), \alpha, \beta>-1$, are orthogonal with respect to the measure $\mu(x)=(1-x)^{\alpha}(1+x)^{\beta}$ on $[-1,1]$. Let $f \in L_{1}(\mu)$, then formally we have 


$$
f(x)=\sum_{k=0}^{\infty} a_{k} h_{k} R_{k}^{(\alpha, \beta)}(x)
$$

where

$$
R_{k}^{(\alpha, \beta)}(x)=\frac{P_{k}^{(\alpha, \beta)}(x)}{P_{k}^{(\alpha, \beta)}(1)}={ }_{2} F_{1}\left(-k, k+\alpha+\beta+1 ; \alpha+1 ; \frac{1-x}{2}\right)
$$

and

$$
a_{k}=\int_{-1}^{1} f(x) R_{k}^{(\alpha, \beta)}(x) d \mu(x), \quad h_{k}^{-1}=\int_{-1}^{1}\left[R_{k}^{(\alpha, \beta)}(x)\right]^{2} d \mu(x) .
$$

Let $f$ be as in (3.1) the translate of $f$ is given (cf. [2]) by

$$
f(x ; y)=\sum_{k=0}^{\infty} a_{k} h_{k} R_{k}^{(\alpha, \beta)}(x) R_{k}^{(\alpha, \beta)}(y) .
$$

This translate is natural in the following sense; if $g(x)$ is expanded similarly with coefficients $b_{k}$ then

$$
(f * g)(x)=(g * f)(x)=\int_{-1}^{1} f(x ; y) g(y) d \mu(y)=\sum_{n=0}^{\infty}\left(a_{k} b_{k}\right) h_{k} R_{k}^{(\alpha, \beta)}(x) .
$$

For a polynomial $K_{n}(x)=\sum_{k=0}^{n} c_{k} h_{k} R_{k}^{(\alpha, \beta)}(x)$, we have

$$
\left(K_{n} * f\right)(x)=\int_{-1}^{1} K_{n}(x ; y) f(y) d \mu(y)=\sum_{k=0}^{\infty}\left(a_{k} c_{k}\right) h_{k} R_{k}^{(\alpha, \beta)}(x) .
$$

We choose [8]

$$
\bar{K}_{n}(x)=t_{n}\left(\frac{1+x}{2}\right)^{n}=t_{n} \sum_{k=0}^{n} c_{k, n} h_{k} R_{k}^{(\alpha, \beta)}(x),
$$

where

$$
t_{n}=\left[\int_{-1}^{1}\left(\frac{1+x}{2}\right)^{n} d \mu(x)\right]^{-1}=\frac{\Gamma(n+\alpha+\beta+2)}{2^{\alpha+\beta+1} \Gamma(n+\beta+1) \Gamma(\alpha+1)} .
$$

The translate of $\bar{K}_{n}(x)$ is

$$
\bar{K}_{n}(x ; y)=t_{n} \sum_{k=0}^{n} c_{k, n} h_{k} R_{k}^{(\alpha, \beta)}(x) R_{k}^{(\alpha, \beta)}(y)
$$

and by a simplification due to Bateman [3] we have

$$
\bar{K}_{n}(x ; y)=(1-x)^{n}(1-y)^{n} \sum_{k=0}^{n} d_{k}\left(\frac{1+x}{1-x}\right)^{k}\left(\frac{1+y}{1-y}\right)^{k},
$$

where

$$
d_{k}=\frac{t_{n}}{4^{n} P_{n}^{(\alpha, \beta)}(1)} \frac{(n-k+\alpha+1)_{k}}{k !} \frac{(k+\beta+1)_{k}}{(n-k) !}>0 .
$$


Note also that [8] $t_{n} c_{k, n} \rightarrow 1$ as $n \rightarrow \infty$ so that the kernels $\bar{K}_{n}(x ; y)$ constitute a positive and finite summability method which is an approximate identity in the sense that the coefficients of $\bar{K}_{n} * f$ converge to those of $f$.

The following theorem, proved first by Horton [8] by different methods, follows now as a special case of Corollary 2.

TheOREM 1. Let $\bar{K}_{n}(x)$ be given as in (3.2) and let $\bar{L}_{n}(x)=\bar{K}_{n}(-x)$. Then

$$
V_{[-1,1]}\left[\bar{K}_{n} * f\right] \leqq Z_{(-1,1)}\left[\bar{K}_{n} * f\right] \leqq V_{[-1,1]}[f]
$$

and

$$
V_{[-1,1]}\left[\bar{L}_{n} * f\right] \leqq Z_{(-1,1)}\left[\bar{L}_{n} * f\right] \leqq V_{[-1,1]}[f]
$$

for all $f \in L_{1}(\mu)$.

As noted in [8] for $\alpha=\beta=-1 / 2$ and $x=\cos \theta$

$$
\bar{K}_{n}(\cos \theta)=\frac{1}{\pi} \frac{(n !)^{2}}{(2 n) !}\left(2 \cos \frac{\theta}{2}\right)^{2 n}
$$

which is the de la Vallée Poussin kernel. Hence, Theorem 1 and more generally Corollary 2 generalize the earlier work of Pólya and Schoenberg [12] for the case $\alpha=\beta=-1 / 2$. Note also that for $\alpha=\beta=-1 / 2$ and $x=\cos \theta$

$$
\bar{L}_{n}(\cos \theta)=\frac{1}{\pi} \frac{(n !)^{2}}{(2 n) !}\left(2 \sin \frac{\theta}{2}\right)^{2 n}
$$

which could be regarded as the adjoint of the de la Vallée Poussin kernel.

4. Kernels of the form $F(z \bar{\zeta})$. Suppose $F(z)$ is analytic in $|z|<\rho, \quad 0<\rho \leqq \infty$ and consider the kernel $K(z, \bar{\zeta})=F(z \bar{\zeta})$ for $|z \bar{\zeta}|<\rho$. Then

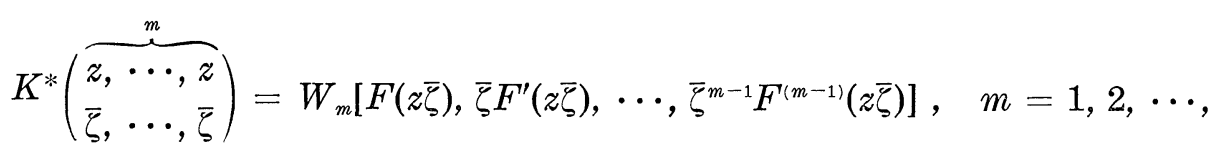

where $W_{m}$ stands for the Wronskian of the $m$ functions with respect to $\bar{\zeta}$. Let $s=z \bar{\zeta}$ then

$$
\begin{aligned}
W_{m} & {\left[F(z \bar{\zeta}), \bar{\zeta} F^{\prime}(z \bar{\zeta}), \cdots, \bar{\zeta}^{m-1} F^{(m-1)}(z \bar{\zeta})\right] } \\
& =\left(\frac{d z}{d \bar{\zeta}}\right)^{m(m-1) / 2} W_{m}\left[F(s), \frac{s}{z} F^{\prime}(s), \cdots,\left(\frac{s}{z}\right)^{m-1} F^{(m-1)}(s)\right] \\
& =W_{m}\left[F(s), s F^{\prime}(s), \cdots, s^{m-1} F^{(m-1)}(s)\right] .
\end{aligned}
$$


Thus

$$
K^{*}(\overbrace{\left.\begin{array}{l}
z, \cdots, z \\
\bar{\zeta}, \cdots, \bar{\zeta}
\end{array}\right)}^{m}=W_{m}\left[F(s), s F^{\prime}(s), \cdots, s^{m-1} F^{(m-1)}(s)\right],
$$

$$
m=1,2, \cdots \text {. }
$$

We say that a sequence of functions $\left\langle f_{m}(z)\right\rangle_{m=1}^{\infty}$, analytic in a domain $D$, constitutes an extended complete Tchebycheff system (ECTsystem) on $D$ if

$$
W_{m}\left[f_{1}(z), \cdots, f_{m}(z)\right] \neq 0, \quad m=1,2, \cdots,
$$

for all $z \in D$.

Consequently, using (4.1), one obtains:

THEOREM 2. Let $F(z)$ be analytic in $|z|<\rho, 0<\rho \leqq \infty$. Then the kernel $K(z, \bar{\zeta})=F(z \bar{\zeta})$ is ESR for $|z \bar{\zeta}|<\rho$ if and only if $\left\langle z^{m-1} F^{(m-1)}(z)\right\rangle_{m=1}^{\infty}$ is ECT on $|z|<\rho$. Especially, if $K(x, y)=F(x y)$ is ESR for $-\rho<x y<\rho$, where $x, y \in \boldsymbol{R}$, then $K(z, \bar{\zeta})=F(z \bar{\zeta})$ is ESR on all the diameters $z \bar{\zeta} \in \boldsymbol{R},|z \bar{\zeta}|<\rho$ and all the $m$ th order signatures are equal.

Several implications of this theorem are immediate. The most trivial one is of course about $F(z)=e^{z}$.

CoRollary 3. The kernel $e_{0}(z, \bar{\zeta})=e^{z \bar{\zeta}}$ is ESR on all $C$ and ETP on all rays passing through the origin (cf. [5] and [9, p. 99]).

Proof. This is because

$$
W_{m}\left[e^{z}, z e^{z}, \cdots, z^{m-1} e^{z}\right]=\left(\prod_{k=1}^{m-1} k !\right) e^{m z}, \quad m=1,2, \cdots
$$

CoROLlaRY 4. The kernel $E_{\alpha}(z, \bar{\zeta})={ }_{1} F_{1}(\alpha ; \alpha+1 ; z \bar{\zeta}), \alpha>0$, is ETP on all rays passing through the origin.

Proof. According to Theorem 2 we have to show that the sequence $x^{m-1} F^{(m-1)}, m=1,2, \cdots$, with $F(x)={ }_{1} F_{1}(\alpha ; \alpha+1 ; x)$ is ECT for $-\infty<x<\infty$. But

$$
F(x)=\frac{\alpha}{x^{\alpha}} \int_{0}^{x} e^{t} t^{\alpha-1} d t, \quad \alpha>0, \quad-\infty<x<\infty .
$$

Therefore 


$$
\begin{aligned}
& W_{m}\left[F(x), x F^{\prime}(x), \cdots, x^{m-1} F^{(m-1)}(x)\right] \\
& \quad=\frac{\alpha^{m}}{x^{\alpha m}} W_{m}\left[G(x), x G^{\prime}(x), \cdots, x^{m-1} G^{(m-1)}(x)\right]
\end{aligned}
$$

with

$$
\begin{array}{r}
G(x)=\int_{0}^{x} e^{t} t^{\alpha-1} d t, \quad G^{(j+1)}(x)=e^{x} x^{\alpha-1} \sum_{k=0}^{j}\left(\begin{array}{l}
j \\
k
\end{array}\right)(-1)^{k}(1-\alpha)_{k} x^{-k}, \\
j=0,1, \cdots .
\end{array}
$$

Consequently

$$
\begin{aligned}
W_{m}\left[G(x), x G^{\prime}(x), \cdots, x^{m-1} G^{(m-1)}(x)\right] & =W_{m}\left[G(x), x^{\alpha} e^{x}, \cdots, x^{\alpha+m-2} e^{x}\right] \\
& =e^{m x} x^{\alpha m} W_{m}\left[H(x), 1, \cdots, x^{m-2}\right] \\
& =e^{m x} x^{\alpha m}\left(\prod_{k=1}^{m-2} k !\right)(-1)^{m-1} H^{(m-1)}(x),
\end{aligned}
$$

where

$$
H(x)=e^{-x} x^{-\alpha} G(x)=e^{-x} x^{-\alpha} \int_{0}^{x} e^{t} t^{\alpha-1} d t=\int_{0}^{1} e^{-x t}(1-t)^{\alpha-1} d t .
$$

Therefore

$$
W_{m}\left[F(x), x F^{\prime}(x), \cdots, x^{m-1} F^{(m-1)}(x)\right]=a^{m}\left(\prod_{k=1}^{m-1} k !\right) e^{m x} \int_{0}^{1} e^{-x t} t^{m-1}(1-t)^{\alpha-1} d t
$$

which is strictly positive for all $-\infty<x<\infty, m=1,2, \cdots$. This concludes the proof.

\section{REMARKS.}

(1) The corollary is in fact a special case of Theorem 6 below.

(2) According to the above proof for $E_{\alpha}(z, \bar{\zeta})={ }_{1} F_{1}(\alpha ; \alpha+1 ; z \bar{\zeta})$, $\alpha>0$,

$$
\begin{aligned}
& E_{\alpha}^{*}(\overbrace{\left.\begin{array}{c}
z, \cdots, z \\
\bar{\zeta}, \cdots, \bar{\zeta}
\end{array}\right)}^{m}=\alpha^{m}\left(\prod_{k=1}^{m-1} k !\right) e^{m z \bar{\zeta}} \int_{0}^{1} e^{-z \bar{\zeta} t} t^{m-1}(1-t)^{\alpha-1} d t, \quad m=1,2, \cdots . \\
& \text { (3) For } \alpha=1, E_{1}(z, \bar{\zeta})=(z \bar{\zeta})^{-1}\left(e^{z \bar{\zeta}}-1\right) \text { and }
\end{aligned}
$$

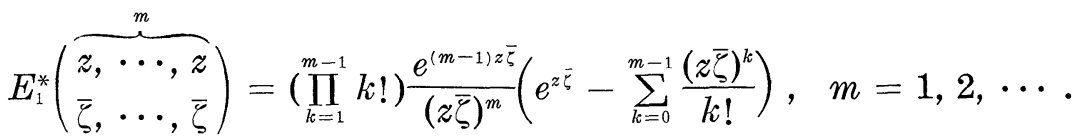

(4) $E_{\alpha}(z, \bar{\zeta})$ is ETP on all rays passing through the origin (i.e., ETP for all $z, \zeta \in \boldsymbol{C}$ with $z \bar{\zeta} \in \boldsymbol{R})$ but it is not ESR on $\boldsymbol{C}$. For example, $E_{1}(2 k \pi i, 1)=0$ for $k=1,2, \cdots$.

Part of the following corollary appears also in [5].

CoROLLARY 5. The kernel $K_{\alpha}(z, \bar{\zeta})=(1-z \bar{\zeta})^{-\alpha}, \alpha \neq 0,-1,-2, \cdots$, 
is ESR on the unit disc $\Delta=\{z:|z|<1\}$. For $\alpha>0$ it is ETP on all diameters of $\Delta$ and for $\alpha<0$ ( $\alpha$ not an integer) it is ESR on the above diameters with the mth order signature

$$
\varepsilon_{m}\left(K_{\alpha}\right)=(-1)^{([-\alpha]+1)(m-1-[-\alpha] / 2)} .
$$

The above is also true when $\zeta$ or $z$ (but not both) lie on the boundary $\partial \Delta=\{z:|z|=1\}$.

Proof. Let $F_{\alpha}(s)=(1-s)^{-\alpha}, \alpha \neq 0,|s|<1$. Then

$$
\begin{aligned}
W_{m} & {\left[F_{\alpha}(s), s F_{\alpha}^{\prime}(s), \cdots, s^{m-1} F_{\alpha}^{(m-1)}(s)\right] } \\
& =W_{m}\left[(1-s)^{-\alpha},(\alpha)_{1} s(1-s)^{-\alpha-1}, \cdots,(\alpha)_{m-1} s^{m-1}(1-s)^{-\alpha-m+1}\right] \\
& =\left(\prod_{k=1}^{m-1}(\alpha)_{k}\right)(1-s)^{-\alpha m} W_{m}\left[1, \frac{s}{1-s}, \cdots,\left(\frac{s}{1-s}\right)^{m-1}\right]
\end{aligned}
$$

and putting $t=s /(1-s)$

$$
\begin{aligned}
& =\left(\prod_{k=1}^{m-1}(\alpha)_{k}\right)(1-s)^{-\alpha m}\left(\frac{d t}{d s}\right)^{m(m-1) / 2} W_{m}\left[1, t, \cdots, t^{m-1}\right] \\
& =\left(\prod_{k=1}^{m-1}(\alpha)_{k} k !\right)(1-s)^{-m(\alpha+m-1)} .
\end{aligned}
$$

Hence $K_{\alpha}(z, \bar{\zeta})$ is ESR for $|z \bar{\zeta}|<1$ and $\alpha \neq 0,-1,-2, \ldots$ On the diameters $z \bar{\zeta} \in \boldsymbol{R}, \quad|z \bar{\zeta}|<1, \quad \varepsilon_{m}\left(K_{\alpha}\right)=\operatorname{sign}\left(\prod_{k=1}^{m-1}(\alpha)_{k}\right)$. Therefore $\varepsilon_{m}\left(K_{\alpha}\right)=+1$ for $\alpha>0$. For $\alpha<0$ ( $\alpha$ not an integer), let $n=[-\alpha]$ that is, $-(n+1)<\alpha<-n$. Thus

$$
\operatorname{sign}\left(\prod_{k=1}^{m-1}(\alpha)_{k}\right)=(-1)^{\sum_{k=1}^{n} k+(n+1)(m-n-1)}=(-1)^{(n+1)(m-1-n / 2)}
$$

and the corollary follows.

CoRollary 6. The kernel $H_{\alpha}(z, \bar{\zeta})=i^{\alpha}(z-\bar{\zeta})^{-\alpha}, \alpha \neq 0,-1,-2, \cdots$, is ESR on the upper half plane $U=\{z: \operatorname{Im} z>0\}$. For $\alpha>0$ it is ETP on all straight lines in $U$ which are parallel to the imaginary axis and for $\alpha<0$ ( $\alpha$ not an integer) it is ESR on the above straight lines with the mth order sigature $\varepsilon_{m}\left(H_{\alpha}\right)=(-1)^{[[-\alpha]+1)(m-1-[-\alpha] / 2)}$. The above is also true when $\zeta$ or $z$ (but not both) lie on the boundary $\partial U=\{z: \operatorname{Im} z=0\}$.

Proof. Under the conformal mapping $w=(z-i) /(z+i) U$ is mapped onto the unit disc $\Delta=\{w:|w|<1\}$ and

$$
H_{\alpha}(z, \bar{\zeta})=i^{\alpha}(z-\bar{\zeta})^{-\alpha}=\left(\frac{d w}{d z}\right)^{\alpha / 2}\left(\overline{\frac{d \tau}{d \zeta}}\right)^{\alpha / 2} K_{\alpha}(w, \bar{\tau}),
$$

where $\tau=(\zeta-i) /(\zeta+i)$ and $K_{\alpha}(w, \bar{\tau})=(1-w \bar{\tau})^{-\alpha}$. Since $d w / d z=$ 
$2 i /(z+i)^{2}$ the corollary follows from Proposition 2 and Corollary 5.

CoRollary 7. The kernel $g_{\alpha}(t, z)=\left(1-2 z t+z^{2}\right)^{-\alpha}, \alpha \neq 0,-1$, $-2, \cdots$, is ESR on $[-1,1] \times \Delta(t \in[-1,1],|z|<1)$. For $\alpha>0$ it is ETP on $[-1,1] \times(-1,1)$ and for $\alpha<0$ ( $\alpha$ not an integer) it is ESR on $[-1,1] \times(-1,1)$ with the $m$ th order signature $\varepsilon_{m}\left(g_{\alpha}\right)=$ $(-1)^{([-\alpha]+1)(m-1-[-\alpha] / 2)}$.

Proof. For

$$
g_{\alpha}(t, z)=\left(1-2 z t+z^{2}\right)^{-\alpha}, \quad(t, z) \in[-1,1] \times \Delta,
$$

we put

$$
\zeta=\frac{1}{2}\left(z+\frac{1}{z}\right), \quad d \zeta / d z=-\frac{1}{2 z^{2}}\left(1-z^{2}\right), \quad z \in \Delta
$$

Then

$$
g_{\alpha}(t, z)=\frac{1}{(2 z)^{\alpha}}(\zeta-t)^{-\alpha}=\frac{1}{(2 i z)^{\alpha}} H_{\alpha}(\zeta, t),
$$

where $H_{\alpha}(\zeta, t)$ is as in Corollary 6. We now use Proposition 2 to obtain

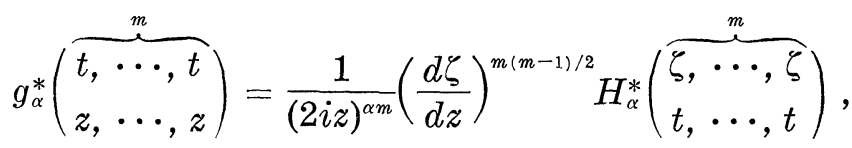

and finally, using Corollary 6 and after some manipulations

$$
\begin{aligned}
& g_{\alpha}^{*}(\overbrace{t, \cdots, t}^{m} \begin{array}{c}
m, \cdots, z
\end{array})=\left(\prod_{k=1}^{m-1}(\alpha)_{k} k !\right)\left[2\left(1-z^{2}\right)\right]^{m(m-1) / 2}\left[g_{\alpha}(t, z)\right]^{m(m-\alpha-1) / \alpha}, \\
& m=1,2, \cdots \text {. }
\end{aligned}
$$

Since $1-2 z t+z^{2}$ does not vanish for $(t, z) \in[-1,1] \times \Delta$ and it is strictly positive for $(t, z) \in[-1,1] \times(-1,1)$ it follows that $g_{\alpha}(t, z)$ is $\operatorname{ESR}$ on $[-1,1] \times \Delta$ for $\alpha \neq 0,-1,-2, \cdots$. For $(t, z) \in[-1,1] \times$ $(-1,1), g_{\alpha}(t, z)$ is ESR with $\varepsilon_{m}\left(g_{\alpha}\right)=\operatorname{sign}\left(\prod_{k=1}^{m-1}(\alpha)_{k}\right)$ and the corollary follows.

5. Reproducing kernels and generating functions. Let $\mathscr{X}$ be a Hilbert space of analytic functions is a domain $D$ with an inner product $(,)_{\varkappa}$. Assume also that $\mathscr{H}$ possesses a reproducing kernel $K(z, \bar{\zeta}),(z, \zeta) \in D \times D$. Thus $f(\zeta)=(f, K(, \bar{\zeta}))_{\mathscr{E}}$ for each $\zeta \in D$ and all $f \in \mathscr{C}$. Let $\left\langle\phi_{n}\right\rangle_{n=0}^{\infty}$ be an orthonormal basis of $\mathscr{H}$. Then [4, p. 98] 


$$
K(z, \bar{\zeta})=\sum_{n=0}^{\infty} \phi_{n}(z) \overline{\phi_{n}(\zeta)}, \quad(z, \zeta) \in D \times D
$$

We set an isometry $T: \mathscr{H} \rightarrow L_{2}(\mu)$ between $\mathscr{C}$ and the Hilbert space $L_{2}(\mu)$. Here $\mu(t)$ is a Borel measure on $\boldsymbol{R}$ with finite moments of all order and with infinitely many points increase. The image of $K(z, \bar{\zeta})$ under this isometry is

$$
g_{T}(t, \bar{\zeta})=\left.T_{z} K(z, \bar{\zeta})\right|_{z=t}, \quad t \in \boldsymbol{R}, \quad \zeta \in D .
$$

(The subscript in $T_{z}$ indicates that $\zeta$ is held fixed and $T$ is applied on $K(z, \bar{\zeta})$ as a function of $z$.) $g_{T}(t, z),(t, z) \in R \times D$, is called the generating function of $\left\langle T \phi_{n}\right\rangle$.

Assume $A \subset \boldsymbol{R}$ is the support of $\mu(t)$. Then, using the reproducing property of $K(z, \bar{\zeta})$, one obtains

$$
K(z, \bar{\zeta})=(K(, \bar{\zeta}), K(, \bar{z}))_{\dddot{r}}=(T K(, \bar{\zeta}), T K(, \bar{z}))_{\mu}
$$

and so

$$
K(z, \bar{\zeta})=\int_{A} g_{T}\left((t, z) g_{T}(t, \bar{\zeta}) d \mu(t), \quad(z, \zeta) \in D \times D\right.
$$

Using (5.1) and Proposition 1, one immediately obtains:

THEOREM 3. Let the assumptions of formula (5.1) prevail and let $\gamma$ be an arc in D. If $g_{T}(t, z)$ is ESR on $A \times \gamma$ then $K(z, \bar{\zeta})$ is ETP on $\gamma$.

Specifically, let $P_{n}(t), n=0,1, \cdots$, be an orthonormal basis of polynomials of degree $n$ in $L_{2}(\mu)$. We write $T \phi_{n}=P_{n}, n=0,1, \cdots$. Since the corresponding elements are orthonormal bases in the spaces $\mathscr{C}$ and $L_{2}(\mu)$ respectively, this correspondence does indeed induce an isometry of $\mathscr{K}$ onto $L_{2}(\mu)$. Therefore

$$
g_{T}(t, z)=\sum_{n=0}^{\infty} P_{n}(t) \phi_{n}(z), \quad(t, z) \in A \times D,
$$

is the required generating function.

THEOREM 4. Let the assumptions of formula (5.2) prevail and let $\gamma$ be an arc in D. If $g_{T}(t, z)$ is ESR on $A \times \gamma$ then $\left\langle\phi_{n}\right\rangle_{n=0}^{\infty}$ is an ECT-system on $\gamma$. In this case $\varepsilon_{m}\left(g_{T}\right)=\varepsilon_{m}(\Phi)$, where $\Phi(n, z)=\phi_{n}(z)$, $(n, z) \in \mathscr{N} \times \gamma, \mathscr{N}=\{0,1,2, \cdots\}$. That is, $\Phi(n, z)$ is ESR on $\mathscr{N} \times \gamma$.

Proof. One has

$$
\begin{aligned}
\Phi(n, z)=\phi_{n}(z) & =\left(\phi_{n}, K(, \bar{z})\right)_{\mathscr{c}}=\left(T \phi_{n}, T K(, \bar{z})\right)_{\mu} \\
& =\left(P_{n}, g_{T}(, \bar{z})\right)_{\mu}=\int_{A} g_{T}(t, z) P_{n}(t) d \mu(t) .
\end{aligned}
$$


Since $\left\langle P_{n}(t)\right\rangle$ is a sequence of polynomials of degree $n,\left\langle P_{n}(t)\right\rangle_{n=0}^{\infty}$ is an ECT-system on $\boldsymbol{R}$. The theorem now follows from Proposition 1.

In the sequel we will show that many reproducing kernels are ESR due to Theorem 3. This, in turn, generalizes our previous results in [5]. We list several examples of this procedure.

6. The Paley-Wiener kernel. The Paley-Wiener space $\mathscr{P}_{a}$ is the space of entire functions $f(z)$ of exponential type $\leqq a$ which belong to $L_{2}$ on the imaginary axis. This is a Hilbert space with the norm

$$
\|f\|^{2}=\int_{-\infty}^{\infty}|f(i y)|^{2} d y .
$$

By a Paley-Wiener theorem [11, p. 13] any $f \in \mathscr{P}_{a}$ is a Laplace transform of a function in $L_{2}(-a, a)$. Thus $T: \mathscr{P}_{a} \rightarrow L_{2}(-a, a)$ given by

$$
\left(T^{-1} g\right)(z)=\frac{1}{2 \pi} \int_{-\infty}^{\infty} e^{t z} g(t) d t \equiv f(z), \quad g \in L_{2}(-a, a),
$$

is an isometry. The reproducing kernel of $\mathscr{P}_{a}$ is

$$
K_{a}(z, \bar{\zeta})=\frac{\sinh (a z+a \bar{\zeta})}{\pi(z+\bar{\zeta})}, \quad z, \zeta \in C
$$

The generating function (the image of $K_{a}(z, \bar{\zeta})$ under $T$ ) is $e^{t z} \chi_{(-a, a)}$, where $\chi_{(-a, a)}$ is the characteristic function of $(-a, a)$. Thus, the following obvious identity

$$
K_{a}(z, \bar{\zeta})=\frac{1}{2 \pi} \int_{-a}^{a} e^{t z} e^{t \overline{\bar{\zeta}}} d t, \quad z, \zeta \in \boldsymbol{C},
$$

holds.

Since $e^{t z}$ is ESR on $\boldsymbol{C} \times \boldsymbol{C}$ (Corollary 3) it follows, by Theorem 3, that $K_{a}(z, \bar{\zeta})$ is ETP on all straight lines in $C$ which are parallel to the real axis. Moreover, $K_{a}(z, \bar{\zeta})$ is ESR on all the strips $\{z:(2 k-1)(\pi / a)<\operatorname{Im} z<(2 k+1)(\pi / a)\}, k=\cdots,-1,0,1, \cdots$.

7. The Hardy kernels. The Hardy space $\mathscr{D}_{q}=\mathscr{D}_{q}(\mathscr{R}), q>0$, is the space of all functions $f(z)$, analytic in the right half plane $\mathscr{R}=\{z: \operatorname{Re} z>0\}$, of the form

$$
f(z)=\int_{0}^{\infty} e^{-z t} F(t) t^{q-(1 / 2)} d t, \quad q>0
$$

with $F \in L_{2}(0, \infty) . \quad \mathscr{D}_{q}$ is a Hibert space with the norm

$$
\|f\|_{q}^{2}=\int_{0}^{\infty}|F(t)|^{2} d t
$$


This Hilbert space has the Hardy reproducing kernel

$$
K_{q}(z, \bar{\zeta})=\Gamma(2 q) /(z+\bar{\zeta})^{2 q}, \quad q>0, \quad z, \zeta \in \mathscr{R} .
$$

The image of $K_{q}(z, \bar{\zeta})$ under the above isometry is $e^{-t \bar{\zeta}}$ and in fact the well-known identity

$$
K_{q}(z, \bar{\zeta})=\int_{0}^{\infty} e^{-t z} e^{-t \bar{\zeta}} t^{q-(1 / 2)} d t, \quad z, \zeta \in \mathscr{R},
$$

holds. Therefore, $K_{q}(z, \bar{\zeta})$ is ESR on $\mathscr{R}$ and ETP on all straight lines in $\mathscr{R}$ which are parallel to the real axis. When $q=1 / 2$ the Hardy kernel reduces to the famous Cauchy kernel $K_{1 / 2}(z, \bar{\zeta})=1 /(z+\bar{\zeta})$. The fact that this kernel is ETP on $(0, \infty)$ is used to establish the well known Müntz theorem on best polynomial approximation.

Clearly, $K_{q}(z, \bar{\zeta})$ and $H_{2 q}(z, \bar{\zeta})$ of Corollary 6 (and for that matter also Corollary 5) are essentially the same (they are melely different realizations of the same object on the conformally equivalent underlying domains $\mathscr{R}$ and $U$ ). In fact let $D$ be a simply-connected domain with the Bergman reproducing kernel, [4, p. 21], $K_{D}(z, \bar{\zeta})$ and let $\varphi: \mathscr{R} \rightarrow D$ be a conformal mapping of $\mathscr{R}$ onto $D$. Let $\psi=\varphi^{-1}$ then $T f=f \circ \psi\left[\psi^{\prime}\right]^{q}$ is an isometry of $\mathscr{D}_{q}(\mathscr{R})$ onto $\mathscr{D}_{q}(D)$. The reproducing kernel of $\mathscr{D}_{q}(D)$ is therefore

$$
K_{q}^{(D)}(z, \bar{\zeta})=\Gamma(2 q) \pi^{q}\left[K_{D}(z, \bar{\zeta})\right]^{q}, \quad q>0, \quad z, \zeta \in D .
$$

Using Corollary 5, Proposition 2 and the above discussion we obtain:

THEOREM 5. Let $D$ be a simply-connected domain with a Bergman reproducing kernel $K_{D}(z, \bar{\zeta})$. Then $\Gamma(2 q) \pi^{q}\left[K_{D}(z, \bar{\zeta})\right]^{q}$ is the reproducing kernel of $\mathscr{D}_{q}(D)$ for $q>0$. Moreover, $\left[K_{D}(z, \bar{\zeta})\right]^{q}$, $2 q \neq 0,-1,0,2, \cdots$, is ESR on $D$. For $q>0$ it is ETP on all arcs $\gamma$ in $D$ which are images of the diameters of the unit disc under the Riemann mapping. For $q<0$ (2q not an integer) $\left[K_{D}(z, \bar{\zeta})\right]^{q}$ is ESR on the above mentioned ares with the mth order signature $\varepsilon_{m}\left(K_{D}^{q}\right)=(-1)^{([-2 q]+1)(m-1-[-2 q] / 2)}$. For $L(z, \bar{\zeta})=\left[K_{D}(z, \bar{\zeta})\right]^{q}$ we have

$$
\begin{aligned}
& L^{*}(\overbrace{\left.\begin{array}{l}
z, \cdots, z \\
\bar{\zeta}, \cdots, \bar{\zeta}
\end{array}\right)}^{m}=\left(\prod_{k=1}^{m-1}(2 q)_{k} k !\right) \pi^{m(m-1) / 2}\left[K_{D}(z, \bar{\zeta})\right]^{m(m+2 q-1) / 2}, \\
& m=1,2, \cdots \text {. }
\end{aligned}
$$

In obtaining the above theorem we made also use of the wellknown fact $\left[4\right.$, p. 33] that if $\varphi: D \rightarrow D^{*}$ is a conformal mapping of $D$ onto $D^{*}$ then

$$
K_{D}(z, \bar{\zeta})=\varphi^{\prime}(z) \overline{\varphi^{\prime}(\zeta)} K_{D} *(\varphi(z), \overline{\varphi(\zeta))}, \quad z, \zeta \in D .
$$


A kernel $K(z, \bar{\zeta})$ which is ESR on $D$ and is of the form $F(z-\bar{\zeta})$ is generally called an extended Pólya frequency (EPF) function on D. Combining (7.1), (7.3) and Theorem 5 we obtain:

Corollary 8. Any power (not of the form $-n / 2, n=0,1, \cdots$ ) of the Bergman kernel is EPF on the right half plane.

Another interesting kernel is obtained by mapping the unit disc $\Delta$ onto the strip $D_{a}=\{z:|\operatorname{Im} z|<a\}$. Since $\phi(z)=\tanh \pi z / 4 a$ maps $D_{a}$ conformally onto $\Delta$ it follows from Theorem 5:

Corollary 9. $\Gamma(2 q)[(\pi / 4 a) \operatorname{sech} \pi(z-\bar{\zeta}) / 4 a]^{2 q}, q>0$, is the reproducing kernel of $\mathscr{D}_{q}\left(D_{a}\right)$. Moreover,

$$
L_{q}(z, \bar{\zeta})=\left[\operatorname{sech} \frac{\pi(z-\bar{\zeta})}{4 a}\right]^{2 q}, \quad 2 q \neq 0,-1, \cdots,
$$

is $\operatorname{ESR}$ on $D_{a}$. For $q>0$ it is $\operatorname{ETP}$ on all arcs $\gamma_{\theta}, 0 \leqq \theta<2 \pi$, given by

$$
z_{\theta}(t)=\frac{2 a}{\pi} \log \frac{1+t e^{i \theta}}{1-t e^{i \theta}}, \quad t \in(-1,1)
$$

For $q<0$ (2q not an integer) it is ESR on the above ares with the $m$ th order signature $\varepsilon_{m}\left(L_{q}\right)=(-1)^{[-2 q]+1)(m-1-[-2 q] / 2)}$. Especially, $L_{q}(x, y), q>0$, is ETP on $(-\infty, \infty) \times(-\infty, \infty)$.

For $q \geqq 1 / 2$ the norm of $\mathscr{D}_{q}$ can be realized in a form which appears frequently in function theory. In fact by another theorem of Paley and Wiener [11, p. 8] one has

$$
\begin{aligned}
\|f\|_{q}^{2} & =\frac{\pi^{-1}}{\Gamma(2 q-1)} \int_{\infty} \int|f(z)|^{2}[2 \operatorname{Re} z]^{2 q-2} d x d y, \quad q>1 / 2, \\
\|f\|_{1 / 2}^{2} & =\frac{1}{2 \pi} \sup _{x>0} \int_{-\infty}^{\infty}|f(x+i y)|^{2} d y .
\end{aligned}
$$

Therefore the norm of $\mathscr{D}_{q}(D), D$ is simply-connected, is given by

$$
\begin{gathered}
\|f\|_{D, q}^{2}=\frac{\pi^{-q}}{\Gamma(2 q-1)} \int_{D} \int|f(z)|^{2} K_{D}(z, \bar{z})^{1-q} d x d y, \quad q>1 / 2 \\
\|f\|_{D, 1 / 2}^{2}=\frac{1}{2 \pi} \int_{\partial D}|f(z)|^{2}|d z|
\end{gathered}
$$

where in the last integral $f$ stands for the nontangential boundary values of the analytic function $f(z)$ in $D$. Consequently, $\mathscr{D}_{q}(D)$, $q>1 / 2$, is in fact the Bergman-Selberg space [14], namely, it is the space of all analytic functions $f(z)$ in $D$ normed by (7.4). This 
Hilbert space possesses the reproducing kernel $K_{q}^{(D)}(z, \bar{\zeta})$ given in (7.2), $q>1 / 2$. Similarly, $\mathscr{D}_{1 / 2}(D)$ is the Szegö space [4, p. 110] consisting of all analytic functions $f(z)$ in $D$ normed by (7.5). Its Szegö reproducing kernel is given by $K_{1 / 2}^{(D)}(z, \bar{\zeta})=\pi^{1 / 2}\left[K_{D}(z, \bar{\zeta})\right]^{1 / 2}$.

These methods can be applied to spaces of square summable series.

8. Spaces of square summable series. Let $D_{\rho}=\{z:|z|<\rho\}$, $0<\rho \leqq \infty$, and assume that $\varphi(z)=\sum_{n=0}^{\infty} c_{n} z^{n}, c_{n}>0$, is analytic in $D_{\rho}$. Let

$$
H_{\varphi}=\left\{f \in H\left(D_{\rho}\right): f(z)=\sum_{n=0}^{\infty} a_{n} z^{n}, \quad \sum_{n=0}^{\infty} \frac{\left|a_{n}\right|^{2}}{c_{n}}<\infty\right\},
$$

where $H\left(D_{\rho}\right)$ designates the class of analytic functions in $D_{\rho}$. Clearly, $H_{\varphi}$ is a Hilbert space with the scalar product

$$
(f, g)=\sum_{n=0}^{\infty} \frac{a_{n} \bar{b}_{n}}{c_{n}} ; \quad\left(f(z)=\sum_{n=0}^{\infty} a_{n} z^{n}, g(z)=\sum_{n=0}^{\infty} b_{n} z^{n}\right) .
$$

We have

$$
f(\zeta)=\sum_{n=0}^{\infty} a_{n} \zeta^{n}=\sum_{n=0}^{\infty} \frac{a_{n} c_{n} \zeta^{n}}{c_{n}}=(f(z), \varphi(z \bar{\zeta})) .
$$

Consequently, $K(z, \bar{\zeta})=\varphi(z \bar{\zeta})$ is the reproducing kernel of $H_{\varphi}$ and $\left\langle\sqrt{c_{n}} z^{n}\right\rangle_{n=0}^{\infty}$ is an orthonormal basis for $H_{\varphi}$ (cf. [15]).

Assume now that $J$ is a real interval and $\mu$ is a finite Borel measure on $J$ with finite moments of all order and with infinitely many points of increase. Consider the Hilbert space $L_{2}(\mu)$ normed by

$$
\|f\|_{\mu^{2}}^{2}=\int_{J}|f(t)|^{2} d \mu(t)
$$

with $P_{n}(t), n=0,1, \cdots$, as an orthonormal basis of polynomials (with real coefficients) of degree $n$. We set up an isometry between $H_{\varphi}$ and $L_{2}(\mu)$ by

$$
T\left(\sqrt{c_{n}} z^{n}\right)=P_{n}(t), \quad n=0,1, \cdots .
$$

The generating function is therefore

$$
g_{T}(t, z)=\sum_{n=0}^{\infty} \sqrt{c_{n}} P_{n}(t) z^{n}, \quad(t, z) \in J \times D_{\rho},
$$

and

$$
K(z, \bar{\zeta})=\varphi(z \bar{\zeta})=\int_{J} g_{T}(t, z) g_{T}(t, \bar{\zeta}) d \mu(t), \quad z, \zeta \in D_{\rho}
$$


Notice that

$$
K(z, \bar{z})=\varphi\left(|z|^{2}\right)=\int_{J}\left[g_{T}(t,|z|)\right]^{2} d \mu(t), \quad z \in D_{\rho} .
$$

Consequently, the question of the total positivity of $K(z, \bar{\zeta})$ on $D_{\rho}$ is reduced, via the above composition formula, to the corresponding problem for $g_{T}(t, z)$ on $J \times D_{\rho}$. Here $g_{T}(t, z)$ is a generating function for certain classical polynomials. We give some examples of this procedure. In these examples we adhere to the convention and standardization of orthogonal polynomials employed in [1, 6, 13].

9. Exponential kernels and Hermite polynomials. Designate by $H(C)$ the class of all entire functions and let

$$
\|f\|_{\alpha}^{2}=\frac{\pi^{-1}}{\Gamma(\alpha+1)} \iint_{C}|f(z)|^{2}|z|^{2 \alpha} e^{-|z|^{2}} d x d y, \quad \alpha>-1
$$

Define

$$
H_{\alpha}=\left\{f \in H(C):\|f\|_{\alpha}<\infty\right\} .
$$

$H_{\alpha}$ is a Hilbert space normed by $\|f\|_{\alpha}$ and in fact

$$
\left\|z^{n}\right\|_{\alpha}^{2}=\frac{1}{\Gamma(\alpha+1)} \int_{0}^{\infty} r^{n+\alpha} e^{-r} d r=(\alpha+1)_{n}, \quad n=0,1, \cdots .
$$

The reproducing kernel of $H_{\alpha}$ is

$$
e_{\alpha}(z, \bar{\zeta})=\sum_{n=0}^{\infty} \frac{(z \bar{\zeta})^{n}}{(\alpha+1)_{n}}={ }_{1} F_{1}(1 ; \alpha+1: z \bar{\zeta}), \quad \alpha>-1, \quad z, \zeta \in C .
$$

Therefore (see Corollary 3)

$$
e_{0}(z, \bar{\zeta})={ }_{1} F_{1}(1 ; 1 ; z \bar{\zeta})=e^{\overline{z \zeta}}
$$

and

$$
e_{\alpha}(z, \bar{\zeta})=\alpha \int_{0}^{1} e^{z \bar{\zeta} t}(1-t)^{\alpha-1} d t, \quad \alpha>0
$$

Especially

$$
e_{1}(z, \bar{\zeta})=\int_{0}^{1} e^{z \bar{\zeta} t} d t=(z \bar{\zeta})^{-1}\left(e^{z \bar{\zeta}}-1\right)
$$

which, according to Corollary 4, is ETP on all rays passing through the origin.

Define now another family of norms by

$$
\|f\|_{\alpha}^{2}=\frac{\pi^{-1}}{\alpha} \iint_{C}|f(z)|^{2}\left(|z|^{2}+\alpha-1\right) e^{-|z|^{2}} d x d y, \quad \alpha>0,
$$


and let

$$
\mathscr{H}_{\alpha}=\left\{f \in H(C):\|f\|_{\alpha}<\infty\right\} .
$$

$\mathscr{H}_{\alpha}$ is also a Hilbert space normed by $\|f\|_{\alpha}$ and in fact

$$
\left\|z^{n}\right\|_{\alpha}^{2}=\alpha^{-1} \int_{0}^{\infty} r^{n}(r+\alpha-1) e^{-r} d r=\alpha^{-1}(\alpha+n) n !, \quad n=0,1, \cdots .
$$

The reproducing kernel of $\mathscr{H}_{\alpha}$ is

$$
E_{\alpha}(z, \bar{\zeta})=\alpha \sum_{n=0}^{\infty} \frac{(z \bar{\zeta})^{n}}{(\alpha+n) n !}={ }_{1} F_{1}(\alpha ; \alpha+1 ; z \bar{\zeta}), \quad \alpha>0, \quad z, \zeta \in C,
$$

and again, according to Corollary 4 , is ETP on all rays passing through the origin. Notice that $E_{1}(z, \bar{\zeta})=e_{1}(z, \bar{\zeta})=(z \bar{\zeta})^{-1}\left(e^{z \bar{\zeta}}-1\right)$. In fact the following relationship

$$
e_{\alpha}(z, \bar{\zeta})=e^{z \bar{\zeta}} E_{\alpha}(-z, \bar{\zeta}), \quad \alpha>0,
$$

between the two families of producing kernels hold.

These kernels are closely connected to the Hermite polynomials. The Hermite polynomials $H_{n}(t)$ are orthogonal with respect to the measure $w(t)=e^{-t^{2}}$ on $(-\infty, \infty)$ and are normalized by

$$
\int_{-\infty}^{\infty}\left[H_{n}(t)\right]^{2} w(t) d t=h_{n}=\sqrt{\pi} 2^{n} n !, \quad n=0,1, \cdots .
$$

In addition they assume the standardization $H_{n}^{(n)}(0)=2^{n} n$ !. We set an isometry $T$ between $H_{0}$ and $L_{2}(w)$ via the rule

$$
T\left(z^{n} / \sqrt{n !}\right)=H_{n}(t) / \sqrt{h_{n}}, \quad n=0,1, \cdots .
$$

The Hermite generating function is therefore

$$
g(t, \bar{\zeta})=T e_{0}(z, \bar{\zeta})=\pi^{-1 / 4} \sum_{n=0}^{\infty} \frac{H_{n}(t)}{n !}\left(2^{-1 / 2 \bar{\zeta}}\right)^{n}
$$

or

$$
g(t, z)=\pi^{-1 / 4} e^{-z^{2} / 2} e^{\sqrt{2} t z}, \quad(t, z) \in \boldsymbol{R} \times \boldsymbol{C} .
$$

Consequently,

$$
e_{0}(z, \bar{\zeta})=e^{z \bar{\zeta}}=\pi^{-1 / 2} e^{-z^{2} / 2-\bar{\zeta}^{2} / 2} \int_{-\infty}^{\infty} e^{\sqrt{2} t(z+\bar{\zeta})} e^{-t^{2}} d t,
$$

which is a well-known identity.

10. Hardy kernels and Laguerre polynomials. The Hardy space $\mathscr{D}_{(\alpha+1) / 2}, \alpha>-1$ (see (7.4)) is defined as the space of all analytic functions in the unit disc $\Delta$ with the moments 


$$
\left\|z^{n}\right\|_{\alpha}^{2}=n ! / \Gamma(\alpha+n+1), \quad \alpha>-1, \quad n=0,1, \cdots .
$$

The norm induced by these moments can be realized as in $\S 7$ and in fact for $\alpha>0$

$$
\|f\|_{\alpha}^{2}=\frac{\pi^{-1}}{\Gamma(\alpha)} \iint_{\Delta}|f(z)|^{2}\left(1-|z|^{2}\right)^{\alpha-1} d x d y
$$

while

$$
\|f\|_{0}^{2}=\frac{1}{2 \pi} \int_{\partial\lrcorner}|f(z)|^{2}|d z|
$$

The reproducing kernel of $H_{\alpha}=\mathscr{D}_{(\alpha+1) / 2}$ is

$$
K(z, \bar{\zeta})=\sum_{n=0}^{\infty} \frac{\Gamma(\alpha+n+1)}{n !}(z \bar{\zeta})^{n}=\Gamma(\alpha+1) /(1-z \bar{\zeta})^{\alpha+1}, \quad z, \zeta \in \Delta .
$$

The fact that these kernels are totally positive is the content of Theorem 5. However, this fact could also be deduced from the Laguerre generating function. The Laguerre polynomials, $L_{n}^{(\alpha)}(t)$, $\alpha>-1$, are orthogonal with respect to the measure $w(t)=e^{-t} t^{\alpha}$ on $[0, \infty)$ and are normalized by

$$
\int_{0}^{\infty}\left[L_{n}^{(\alpha)}(t)\right]^{2} w(t) d t=h_{n}=\Gamma(\alpha+n+1) / n !, \quad n=0,1, \cdots .
$$

We assume also the standardization $d^{n} / d t^{n} L_{n}^{(\alpha)}(0)=(-1)^{n}$. Clearly, $T: H_{\alpha} \rightarrow L_{2}(w)$ given by $T\left(\sqrt{h_{n}} z^{n}\right)=L_{n}^{(\alpha)}(t) / \sqrt{h_{n}}$ is an isometry of $H_{\alpha}$ onto $L_{2}(w)$. The Laguerre generating function is therefore

$$
g(t, z)=\sum_{n=0}^{\infty} L_{n}^{(\alpha)}(t) z^{n}=(1-z)^{-\alpha-1} e^{-t z /(1-z)}, \quad(t, z) \in[0, \infty) \times \Delta .
$$

Hence

$$
K_{\alpha}(z, \bar{\zeta})=\frac{\Gamma(\alpha+1)}{(1-z \bar{\zeta})^{\alpha+1}}=(1-z)^{-\alpha-1}(1-\bar{\zeta})^{-\alpha-1} \int_{0}^{\infty} e^{-t \bar{\zeta} /(1-\bar{\zeta})} e^{-t z /(1-z)} w(t) d t,
$$

which is, according to Propositions 1, 2 and Corollary 3, the required result.

11. Logarithmic kernels and Laguerre polynomials. Consider the unit disc $\Delta$ and let the moments be given by

$$
\left\|z^{n}\right\|_{\alpha}^{2}=\frac{(\alpha+n)^{2}}{\alpha^{2}} \frac{n !}{\Gamma(\alpha+n+1)}, \quad \alpha>0, \quad n=0,1, \cdots .
$$

With (11.1) we have a Hilbert space $\mathscr{H}_{\alpha}$ of analytic functions $f(z)=$ $\sum_{n=0}^{\infty} a_{n} z^{n}$ in $\Delta$. The reproducing kernel of $\mathscr{H}_{\alpha}$ is given by 


$$
K_{\alpha}(z, \bar{\zeta})=a^{2} \sum_{n=0}^{\infty} \frac{\Gamma(\alpha+n+1)}{(\alpha+n)^{2}} \frac{(z \bar{\zeta})^{n}}{n !}=\Gamma(\alpha+1)_{2} F_{1}(\alpha, \alpha ; \alpha+1 ; z \bar{\zeta}),
$$

for $z, \zeta \in \Delta \cdot T: \mathscr{H}_{\alpha} \rightarrow L_{2}(w)$ given by $T\left(\sqrt{c_{n}} z^{n}\right)=L_{n}^{(\alpha)}(t) / \sqrt{h_{n}}$, where $c_{n}^{-1}=\left\|z^{n}\right\|_{\alpha}^{2}$ and $h_{n}=\Gamma(\alpha+n+1) / n !, n=0,1, \cdots$, is an isometry of $\mathscr{C}_{\alpha}$ onto $L_{2}(w)$. Here, as before, $w(t)=e^{-t} t^{\alpha}$ on $[0, \infty)$. The generating function (cf. [13, p. 202]) is therefore

$$
\begin{aligned}
G_{\alpha}(t, \bar{\zeta}) & =T K_{\alpha}(z, \bar{\zeta})=\sum_{n=0}^{\infty} \frac{(a)_{n}}{(\alpha+1)_{n}} L_{n}^{(\alpha)}(t) \bar{\zeta}^{n} \\
& =(1-\bar{\zeta})^{-\alpha}{ }_{1} F_{1}\left(\alpha ; \alpha+1 ;-\frac{t \bar{\zeta}}{1-\bar{\zeta}}\right) .
\end{aligned}
$$

Thus

$$
\begin{aligned}
K_{\alpha}(z, \bar{\zeta})= & (1-z)^{-\alpha}(1-\bar{\zeta})^{-\alpha} \\
& \times \int_{0}^{\infty}{ }_{1} F_{1}\left(\alpha ; \alpha+1 ;-\frac{t z}{1-z}\right)_{1} F_{1}\left(\alpha ; \alpha+1 ;-\frac{t \bar{\zeta}}{1-\bar{\zeta}}\right) w(t) d t .
\end{aligned}
$$

Since, according to Proposition 2 and Corollary $4, G_{\alpha}(t, z)$ is ETP on $[0, \infty) \times(-1,1)$ it follows that $K_{\alpha}(z, \bar{\zeta})$ is ESR on $\Delta$ and ETP on all diameters of $\Delta$.

An interesting special case occurs when $\alpha=1$. In this case $\mathscr{H}_{1}$ is the Dirichlet space consisting of all analytic functions in the unit dise $\Delta$ normed by

$$
\|f\|_{1}^{2}=\frac{1}{\pi} \iint_{\Delta}\left|f^{\prime}(z)\right|^{2} d x d y+\frac{1}{2 \pi} \int_{\partial \Delta}|f(z)|^{2}|d z| .
$$

where $f$ in the second integral stands for the nontangential boundary values of the analytic function $f(z)$ in $\Delta$. $\mathscr{H}_{1}$ has the logarithmic reproducing kernel

$$
K_{1}(z, \bar{\zeta})=-(z \bar{\zeta})^{-1} \log (1-z \bar{\zeta}), \quad z, \zeta \in \Delta .
$$

The same applies when $\alpha=2$. In this case

$$
K_{2}(z, \bar{\zeta})=\frac{4}{z \bar{\zeta}}\left[(1-z \bar{\zeta})^{-1}+(z \bar{\zeta})^{-1} \log (1-z \bar{\zeta})\right], \quad z, \zeta \in \Delta
$$

As stated before both kernels are ETP on all diameters of $\Delta$. We should remark however that actually not only ${ }_{2} F_{1}(\alpha, \alpha ; \alpha+1 ; z \bar{\zeta})$, $\alpha>0$, is ETP on the diameters of $\Delta$ but also ${ }_{2} F_{1}(\alpha, \beta ; \alpha+1 ; z \bar{\zeta})$, $\alpha, \beta>0$. This will be shown as a special case of Theorem 7 .

12. Tchebycheff kernels. We now treat three different Hilbert spaces, arising from the Tchebycheff polynomials, all of which have ESR reproducing kernels. 
Designate by $H_{1}$ the Hilbert space of analytic functions in the unit disc $\Delta$ with the scalar product

$$
(f, g)_{1}=\frac{1}{4 \pi^{2}}\left[\int_{\partial \Delta} f(z) \overline{g(z)}|d z|+2 \pi f(0) \overline{g(0)}\right], \quad f, g \in H_{1},
$$

where, as usual, the integral is carried over the nontangential boundary values of $f$ and $g$. Here, of course,

$$
H_{1}=\left\{f \in H(\Delta):\|f\|_{1}^{2}=(f, f)_{1}<\infty\right\},
$$

and

$$
\left(z^{n}, z^{m}\right)_{1}=\frac{1}{2 \pi}\left[\delta_{n, m}+\delta_{0, n} \delta_{0, m}\right],
$$

where $\delta_{n, m}$ stands for the Delta of Kronecker for $n$ and $m$. Consequently, $\sqrt{c_{n}} z^{n}, n=0,1, \cdots$, with $c_{0}=\pi$ and $c_{n}=2 \pi$ for $n \geqq 1$, is an orthonormal basis for $H_{1}$. The reproducing kernel is

$$
K_{1}(x, \bar{\zeta})=\pi+2 \pi \sum_{n=1}^{\infty}(z \bar{\zeta})^{n}=\pi \frac{1+z \bar{\zeta}}{1-z \bar{\zeta}}, \quad z, \zeta \in \Delta .
$$

We will now show that $K_{1}(z, \bar{\zeta})$ is ESR on $\Delta$.

The Tchebycheff polynomials (of the first kind) $T_{n}(t)$ are orthogonal with respect to the measure $w(t)=\left(1-t^{2}\right)^{-1 / 2}$ on $[-1,1]$ and are normalized by

$$
\int_{-1}^{1}\left[T_{n}(t)\right]^{2} w(t) d t=h_{n}=\frac{\pi}{2}\left(1+\delta_{n, 0}\right), \quad n=0,1, \cdots .
$$

We assume also the standardization $T_{n}(1)=1$. $\quad T: H_{1} \rightarrow L_{2}(w)$ given by $T\left(\sqrt{c_{n}} z^{n}\right)=T_{n}(t) / \sqrt{h_{n}}, n=0,1, \cdots$, is an isometry of $H_{1}$ onto $L_{2}(w)$. The generating function (cf. [7, p. 186]) is

$$
g(t, \bar{\zeta})=T K_{1}(z, \bar{\zeta})=\sum_{n=0}^{\infty}\left(2-\delta_{n, 0}\right) T_{n}(t) \bar{\zeta}^{n}=\left(1-\bar{\zeta}^{2}\right) g_{1}(t, \bar{\zeta})
$$

where $g_{1}(t, z)$ is as in $(4.2)$

$$
g_{1}(t, z)=\left(1-2 z t+z^{2}\right)^{-1}, \quad(t, z) \in[-1,1] \times \Delta .
$$

Consequently,

$$
K_{1}(z, \bar{\zeta})=\left(1-z^{2}\right)\left(1-\bar{\zeta}^{2}\right) \int_{-1}^{1} g_{1}(t, z) g_{1}(t, \bar{\zeta}) w(t) d t, \quad z, \zeta \in \Delta .
$$

It follows, using Corollary 7 , that $K_{1}(z, \bar{\zeta})$ is ESR on $\Delta$ and ETP on all diameters $\Delta$.

Let $H_{2}=\left\{f \in H(\Delta):\|f\|_{2}<\infty\right\}$, where 


$$
\|f\|_{2}^{2}=\frac{1}{8 \pi^{2}}\left[\int_{\partial\lrcorner}|f(z)|^{2}|d z|-\pi|f(0)|^{2}\right]
$$

This is clearly a norm for, $\pi|f(0)|^{2} \leqq 2 \pi|f(0)|^{2} \leqq \int_{\partial}|f(z)|^{2}|d z|$. (The integral as usual is carried over the nontangential boundary values of $f) . \quad H_{2}$ is a Hilbert space with the scalar product

$$
(f, g)_{2}=\frac{1}{8 \pi^{2}} \int_{\partial \mid}\left(f(z) \overline{g(z)}-\frac{1}{2} f(0) \overline{g(0))}|d z|\right.
$$

and

$$
\left\|z^{n}\right\|_{2}^{2}=c_{n}^{-1}=\frac{1}{4 \pi}\left(1-\frac{1}{2} \delta_{n, 0}\right) .
$$

The reproducing kernel is

$$
K_{2}(z, \bar{\zeta})=8 \pi+4 \pi \sum_{n=1}^{\infty}(z \bar{\zeta})^{n}=4 \pi \frac{2-z \bar{\zeta}}{1-z \bar{\zeta}}, \quad z, \zeta \in \Delta
$$

This kernel is also ESR on $\Delta$. For this we introduce (cf. [1, p. 774]) the modified Tchebycheff polynomials (of the first kind) $S_{n}(t)$. These polynomials are orthogonal with respect to $\mu(t)=\left(1-t^{2} / 4\right)^{-1 / 2}$ on $[-2,2]$ and normalized by

$$
\int_{-2}^{2}\left[S_{n}(t)\right]^{2} \mu(t) d t=h_{n}=4 \pi\left(1+\delta_{n, 0}\right), \quad n=0,1, \cdots
$$

In addition $S_{n}(t)$ assumes the standardization $S_{n}(2)=n+1$. In this case the generating function is given by $[1, p .784]$

$$
G(t, z)=\sum_{n=0}^{\infty} S_{n}(t) z^{n}=\left(1-z t+z^{2}\right)^{-1}=g_{1}(t / 2, z),
$$

where $(t, z) \in[-2,2] \times \Delta$. Here $h_{n}=c_{n}$ and $T: H_{2} \rightarrow L_{2}(\mu)$ given by $T\left(\sqrt{c_{n}} z^{n}\right)=S_{n}(t) / \sqrt{c_{n}}$ is an isometry of $H_{2}$ onto $L_{2}(\mu)$. Therefore

$$
T K_{2}(z, \bar{\zeta})=\sum_{n=0}^{\infty} S_{n}(t) \bar{\zeta}^{n}=g_{1}(t / 2, \bar{\zeta}), \quad(t, \zeta) \in[-2,2] \times \Delta .
$$

But according to Corollary $7, g_{1}(t / 2, z)$ is $\operatorname{ESR}$ on $[-2,2] \times \Delta$ whence $K_{2}(z, \bar{\zeta})$ is ESR on $\Delta$ and ETP on all diameters of $\Delta$.

We now introduce a third Hilbert space $H_{3}$ of analytic functions in the unit disc $\Delta . H_{3}$ is normed via the moments

$$
\left\|z^{n}\right\|_{3}^{2}=c_{n}^{-1}=\frac{1}{4 \pi}\left((n+1)^{-2}+\delta_{n, 0}\right) .
$$

The reproducing kernel of $H_{3}$ is therefore 


$$
K_{3}(z, \bar{\zeta})=8 \pi+4 \pi \sum_{n=1}^{\infty}(n+1)^{2}(z \bar{\zeta})^{n}=4 \pi\left[1+\frac{1+z \bar{\zeta}}{(1-z \bar{\zeta})^{3}}\right], \quad z, \zeta \in \Delta
$$

In order to prove that $K_{3}(z, \bar{\zeta})$ is $\operatorname{ESR}$ on $\Delta$ we differentiate $z G(t, z)$ with respect to $z$, where $G(t, z)$ is given by (12.2). We obtain

$(z G(t, z))^{\prime}=\sum_{n=0}^{\infty}(n+1) S_{n}(t) z^{n}=\left(1-z^{2}\right)\left(1-z t+z^{2}\right)^{-2}=\left(1-z^{2}\right) g_{2}(t / 2, z)$,

where $(t, z) \in[-2,2] \times \Delta$ and $g_{2}(t / 2, z)$ is as in (4.2). According to Corollary 7, $\left(1-z^{2}\right) g_{2}(t / 2, z)$ is ESR on $[-2,2] \times \Delta$ and ETP on $[-2,2] \times(-1,1)$. The natural isometry $T: H_{3} \rightarrow L_{2}(\mu)$ given by $\left(T \sqrt{c_{n}} z^{n}\right)=S_{n}(t) / \sqrt{h_{n}}$, yields

$$
T K_{3}(z, \bar{\zeta})=\sum_{n=0}^{\infty}\left(\frac{c_{n}}{h_{n}}\right)^{1 / 2} S_{n}(t) \bar{\zeta}^{n}=\sum_{n=0}^{\infty}(n+1) S_{n}(t) \bar{\zeta}^{n}=\left(1-\bar{\zeta}^{2}\right) g_{2}(t / 2, \bar{\zeta})
$$

and

$$
K_{3}(z, \bar{\zeta})=\left(1-z^{2}\right)\left(1-\bar{\zeta}^{2}\right) \int_{-2}^{2} g_{2}(t / 2, z) g_{2}(t / 2, \bar{\zeta}) \mu(t) d t
$$

Consequently $K_{3}(z, \bar{\zeta})$ is ESR on $\Delta$ and ETP on the diameters of $\Delta$.

13. Gegenbauer kernels. Let $H_{\alpha}, 0 \neq \alpha>-1 / 2$, be the Hilbert space of analytic functions in the unit disc $\Delta$ which is normed via

$$
\left\|z^{n}\right\|_{\alpha}^{2}=h_{n}^{-1}=\frac{[\Gamma(\alpha)]^{2}}{\pi 2^{1-2 \alpha}} \frac{n !(n+\alpha)}{\Gamma(n+2 \alpha)}, \quad n=0,1, \cdots .
$$

The reproducing kernel of $H_{\alpha}$ is

$$
\begin{aligned}
K_{\alpha}(z, \bar{\zeta}) & =\sum_{n=0}^{\infty} h_{n}(z \bar{\zeta})^{n} \\
& =B(1 / 2, \alpha+1 / 2)_{2} F_{1}(2 \alpha, \alpha ; \alpha+1 ; z \bar{\zeta}), \quad z, \zeta \in \Delta .
\end{aligned}
$$

Here $B(p, q)$ is the Beta function and in obtaining (13.1) we made use of the duplication formula

$$
\sqrt{\pi} \Gamma(2 \alpha)=2^{2 \alpha-1} \Gamma(\alpha) \Gamma(\alpha+1 / 2) .
$$

The Gegenbauer polynomials $C_{n}^{(\alpha)}(t)$ are orthogonal with respect to the measure $w(t)=\left(1-t^{2}\right)^{\alpha-1 / 2}, \quad 0 \neq \alpha>-1 / 2$, on $[-1,1]$ and normalized by

$$
\int_{-1}^{1}\left[C_{n}^{(\alpha)}(t)\right]^{2} w(t) d t=h_{n}, \quad n=0,1, \cdots .
$$

They assume the standardization $C_{n}^{(\alpha)}(1)=(2 \alpha)_{n} / n !$. We again set an isometry $T: H_{\alpha} \rightarrow L_{2}(w)$ by the rule $T\left(\sqrt{h_{n}} z^{n}\right)=C_{n}^{(\alpha)}(t) / \sqrt{h_{n}}$ so that the generating function is 


$$
T K_{\alpha}(z, \bar{\zeta})=\sum_{n=0}^{\infty} C_{n}^{(\alpha)}(t) \bar{\zeta}^{n}=\left(1-2 \bar{\zeta} t+\bar{\zeta}^{2}\right)^{-\alpha}=g_{\alpha}(t, \bar{\zeta})
$$

and so

$$
K_{\alpha}(z, \bar{\zeta})=\int_{-1}^{1} g_{\alpha}(t, z) g_{\alpha}(t, \bar{\zeta}) w(t) d t
$$

Since $\alpha \neq 0,-1,-2, \cdots$, it follows from Corollary 7 that $K_{\alpha}(z, \bar{\zeta})$ is ESR on $\Delta$ and ETP on the diameters of $\Delta$.

For $\alpha>0, K_{\alpha}(z, \bar{\zeta})$ admits various integral representations namely,

$$
K_{\alpha}(z, \bar{\zeta})=\alpha B(1 / 2, \alpha+1 / 2) \int_{0}^{1} t^{\alpha-1}(1-t z \bar{\zeta})^{-2 \alpha} d t, \quad \alpha>0 .
$$

If in addition $\alpha<1$ then

$$
K_{\alpha}(z, \bar{\zeta})=2^{1-2 \alpha} \sin (\alpha \pi) \int_{0}^{1} t^{2 \alpha-1}(1-t)^{-\alpha}(1-t z \bar{\zeta})^{-\alpha} d t, \quad 0<\alpha<1 .
$$

The special case when $\alpha=1$ is well known. In this case $C_{n}^{(1)}(t)=U_{n}(t)$ are the Tchebycheff polynomials (of the second kind) and $\left\|z^{n}\right\|_{1}^{2}=2 / \pi$. Then norm of $H_{1}$ is realized via

$$
\|f\|_{1}^{2}=\pi^{-2} \int_{\partial\lrcorner}|f(z)|^{2}|d z|, \quad f \in H_{1} .
$$

$H_{1}$ is therefore the Szegö space with the Szegö kernel

$$
K_{1}(z, \bar{\zeta})=\frac{\pi}{2}(1-z \bar{\zeta})^{-1}, \quad z, \zeta \in \Delta
$$

Another special case is when $\alpha=1 / 2$. In this case $C_{n}^{(1 / 2)}(t)=P_{n}(t)$ are the Legendre polynomials and $\left\|z^{n}\right\|_{1 / 2}^{2}=n+1 / 2$. The norm of $H_{1 / 2}$ is realized via

$$
\|f\|_{1 / 2}^{2}=\frac{1}{\pi} \iint_{\Delta}\left|\left[z^{1 / 2} f(z)\right]^{\prime}\right|^{2} d x d y, \quad f \in H_{1 / 2} .
$$

The space $H_{1 / 2}$ is essentially the Dirichlet space $\mathscr{H}_{1}$ introduced in (11.2). In fact, it is easily verified that $2^{-1}\|f\|_{1}^{2} \leqq\|f\|_{1 / 2}^{2} \leqq\|f\|_{1}^{2}$ where $\|f\|_{1}^{2}$ is as in (11.2). Moreover,

$$
K_{1 / 2}(z, \bar{\zeta})=\frac{1}{\sqrt{z \bar{\zeta}}} \log \frac{1+\sqrt{z \bar{\zeta}}}{1-\sqrt{z \bar{\zeta}}}=\frac{2}{\sqrt{z \bar{\zeta}}} \tanh ^{-1} \sqrt{z \bar{\zeta}}, \quad z, \zeta \in \Delta,
$$

and it should be compared to the other ESR reproducing kernel in (11.3). Note that $K_{1 / 2}(z, \bar{z})=2 \rho_{N}(0, z) /|z|$ where $\rho_{N}(0, z)$ is the hyperbolic distance of 0 to $z$ in the unit disc $\Delta$. Therefore 
$|w| K_{1 / 2}(w, \bar{w}) \leqq|z| K_{1 / 2}(z, \bar{z})$ for any analytic mapping $w=\varphi(z)$ of $\Delta$ into itself and $\varphi(0)=0$. Equality holds if and only if $\varphi(z)=e^{i \theta} z$. Using the reproducing property of $K_{1 / 2}(z, \bar{\zeta})$ one obtains

$$
K_{1 / 2}(\zeta, \bar{\zeta})=\frac{1}{\pi} \iint_{1}|1-z \bar{\zeta}|^{-2}|z|^{-1} d x d y
$$

We now consider another family of Hilbert spaces $\mathscr{H}_{\alpha}$. $\mathscr{H}_{\alpha}$, $0 \neq \alpha>-1 / 2$, is the space of analytic functions in the unit disc $\Delta$ normed by

$$
\left\|z^{n}\right\|_{\alpha}^{2}=c_{n}^{-1}=\frac{[\Gamma(\alpha+1)]^{2}}{\pi 2^{1-2 \alpha}} \frac{n !}{(n+\alpha) \Gamma(n+2 \alpha)}, \quad n=0,1, \cdots .
$$

The reproducing kernel of $\mathscr{C}_{\alpha}$ is

$$
\mathscr{K}_{\alpha}(z, \bar{\zeta})=B(1 / 2, \alpha+1 / 2)_{2} F_{1}(2 \alpha, \alpha+1 ; \alpha ; z \bar{\zeta}), \quad z, \zeta \in \Delta .
$$

Here, again, we have used the duplication formula. According to [6, p. 101]

$$
\mathscr{K}_{\alpha}(z, \bar{\zeta})=B(1 / 2, \alpha+1 / 2) \frac{1+z \bar{\zeta}}{(1-z \bar{\zeta})^{2 \alpha+1}}, \quad z, \zeta \in \Delta .
$$

Though (13.3) is not defined for $\alpha=0$ we can actually incorporate this value in (13.4). In fact $\mathscr{K}_{0}(z, \bar{\zeta})$ is exactly $K_{1}(z, \bar{\zeta})$ defined in (12.1). $\mathscr{K}_{0}(z, \bar{\zeta})$ is therefore ESR on $\Delta$. For $\alpha \neq 0$ we set the isometry $T: \mathscr{C}_{\alpha} \rightarrow L_{2}(w)$ by the rule $T\left(\sqrt{c_{n}} z^{n}\right)=C_{n}^{(\alpha)}(t) / \sqrt{h_{n}}$ so that the generating function is

$$
T \mathscr{K}_{\alpha}(z, \bar{\zeta})=\sum_{n=0}^{\infty}\left(\frac{c_{n}}{h_{n}}\right)^{1 / 2} C_{n}^{(\alpha)}(t) \bar{\zeta}^{n}=\sum_{n=0}^{\infty}\left(\frac{n+\alpha}{\alpha}\right) C_{n}^{(\alpha)}(t) \bar{\zeta}^{n} .
$$

This generating function admits a closed form expression. Indeed, by differentiating the identity

$$
z^{\alpha} \sum_{n=0}^{\infty} C_{n}^{(\alpha)}(t) z^{n}=\left[z\left(1-2 z t+z^{2}\right)^{-1}\right]^{\alpha}, \quad 0 \neq \alpha>-1 / 2,
$$

with respect to $z$, we obtain

$$
\sum_{n=0}^{\infty}\left(\frac{n+\alpha}{\alpha}\right) C_{n}^{(\alpha)}(t) z^{n}=\left(1-z^{2}\right)\left(1-2 z t+z^{2}\right)^{-(\alpha+1)}=\left(1-z^{2}\right) g_{\alpha+1}(t, z) .
$$

Therefore $T \mathscr{K}_{\alpha}(z, \bar{\zeta})=\left(1-\bar{\zeta}^{2}\right) g_{\alpha+1}(t, \bar{\zeta})$ and

$$
\begin{aligned}
\mathscr{K}_{\alpha}(z, \bar{\zeta}) & =B(1 / 2, \alpha+1 / 2) \frac{1+z \bar{\zeta}}{(1-z \bar{\zeta})^{2 \alpha+1}} \\
& =\left(1-z^{2}\right)\left(1-\bar{\zeta}^{2}\right) \int_{-1}^{1} g_{\alpha+1}(t, z) g_{\alpha+1}(t, \bar{\zeta}) w(t) d t
\end{aligned}
$$


is in fact true for all $\alpha>-1 / 2$ (including $\alpha=0$ ). Consequently, using Corollary $7, \mathscr{K}_{\alpha}(z, \bar{\zeta})$ is ESR on $\Delta$ and ETP on all diameters of 4 .

Since $\int_{-1}^{1} w(t) d t=\int_{-1}^{1}\left(1-t^{2}\right)^{\alpha-1 / 2} d t=B(1 / 2, \alpha+1 / 2)$ by reasons of continuity Corollary 7 coupled with (13.5) actually yields a stronger result, namely, that

$$
{ }_{2} F_{1}(2 \alpha, \alpha+1 ; \alpha ; z \bar{\zeta})=(1+z \bar{\zeta})(1-z \bar{\zeta})^{-(2 \alpha+1)}, \quad z, \zeta \in \Delta,
$$

is ESR on $\Delta$ and ETP on the diameters of $\Delta$ for all $\alpha, \alpha \neq-n / 2$, $n=1,2, \cdots$.

We again take two special cases $\alpha=1 / 2$ and $\alpha=1$ (corresponding to Legendre and Tchebycheff (second kind) polynomials respectively). For $\alpha=1 / 2$ the moments are $\left\|z^{n}\right\|_{1 / 2}^{2}=2^{-1}(2 n+1)^{-1}$. The norm of $\mathscr{H}_{1 / 2}$ is realized through

$$
\|f\|_{1 / 2}^{2}=(4 \pi)^{-1} \iint_{1}\left|z^{-1 / 2} f(z)\right|^{2} d x d y, \quad f \in \mathscr{K}_{1 / 2},
$$

and $\mathscr{K}_{1 / 2}(z, \bar{\zeta})=2(1+z \bar{\zeta})(1-z \bar{\zeta})^{-2}$. For $\alpha=1$ the moments are $\left\|z^{n}\right\|_{1}^{2}=2 \pi^{-1}(n+1)^{2}$ and the norm of $\mathscr{H}_{1}$ is

$$
\|f\|_{1}^{2}=\pi^{-2} \int_{\partial !}|F(z)|^{2}|d z|, \quad f \in \mathscr{H}_{1},
$$

where $F^{\prime}(z)=z f(z)$ and the integral is carried over the nontangential boundary values of $F(z)$. Here $\mathscr{K}_{1}(z, \bar{\zeta})=2^{-1} \pi(1+z \bar{\zeta})(1-z \bar{\zeta})^{-3}$.

14. Generalized hypergeometric kernels. The previous results can be generalized to include kernels formed from the generalized hypergeometric functions. The generalized hypergeometric function $[13$, pp. $73-85]$ is defined by

$$
{ }_{p} F_{q}\left[\begin{array}{l}
\alpha_{1}^{\prime}, \cdots, \alpha_{p} ; z \\
\beta_{1}, \cdots, \beta_{q} ;
\end{array}\right]=1+\sum_{n=1}^{\infty} \frac{A_{n}(p)}{B_{n}(q)} \frac{z^{n}}{n !},
$$

where

$$
A_{n}(p)=\prod_{j=1}^{p}\left(\alpha_{j}\right)_{n}, \quad B_{n}(q)=\prod_{j=1}^{q}\left(\beta_{j}\right)_{n}, \quad n=1,2, \cdots
$$

Here $\beta_{j} \notin\{0,-1,-2, \cdots\}, j=1,2, \cdots, q$. We exclude the polynomial case, that is, when $\alpha_{j}=0$ for some $j, 1 \leqq j \leqq p$. In this case the series in (14.1) diverges for $z \neq 0$ if $p>q+1$ and we therefore only consider the case in which $p \leqq q+1$. If $p \leqq q$ the series in (14.1) represents an entire function while if $p=q+1$ it is an analytic function in the unit disc $\Delta$. In the notation of (14.1) we have

$$
{ }_{0} F_{0}(-;-; z)=e^{z}, \quad{ }_{1} F_{0}(\alpha ;-; z)=(1-z)^{-\alpha} .
$$


If $A_{n}(p) / B_{n}(q)>0, n=1,2, \cdots$, then

$$
K(z, \bar{\zeta})={ }_{p} F_{q}\left[\begin{array}{l}
\alpha_{1}, \cdots, \alpha_{p} ; z \bar{\zeta} \\
\beta_{1}, \cdots, \beta_{q} ;
\end{array}\right]
$$

is the reproducing kernel of the Hilbert space of analytic functions (in $C$ if $p \leqq q$ and in $\Delta$ if $p=q+1$ ) $f(z)=\sum_{n=0}^{\infty} a_{n} z^{n}$ normed by

$$
\|f\|^{2}=\sum_{n=0}^{\infty} n ! \frac{B_{n}(q)}{A_{n}(p)}\left|a_{n}\right|^{2} .
$$

The generalized hypergeometric functions admit the following $[13$, p. 85] integral representation;

$$
\begin{aligned}
& { }_{p} F_{q}\left[\begin{array}{l}
\alpha_{1}, \alpha_{2}, \cdots, \alpha_{p} ; \\
\beta_{1}, \beta_{2}, \cdots, \beta_{q} ;
\end{array}\right] \\
& =\frac{1}{B\left(\alpha_{1}, \beta_{1}-\alpha_{1}\right)} \int_{0}^{1} t^{\alpha_{1}-1}(1-t)^{\beta_{1}-\alpha_{1}-1}{ }_{p-1} F_{q-1}\left[\begin{array}{l}
\alpha_{2}, \cdots, \alpha_{p} ; \\
\beta_{2}, \cdots, \beta_{q} ;
\end{array}\right] d t,
\end{aligned}
$$

where $\operatorname{Re} \beta_{1}>\operatorname{Re} \alpha_{1}>0$. For $p \leqq q$ (14.3) holds for all $z \in C$ and for $p=q+1$ (14.3) holds for all $z \in \Delta$.

We now prove a generalization of Corollary 4 .

THEOREM 6. $F_{q}(z \bar{\zeta})={ }_{q} F_{q}\left[\begin{array}{l}\alpha_{1} \\ \alpha_{1}+1, \cdots, \alpha_{q}\end{array} ; z \bar{\zeta}\right], \alpha_{j}>0, j=1$, $\cdots, q$, is the reproducing kernel of the Hilbert space of entire functions normed by (14.2) and is $\mathrm{ETP}$ on all rays passing through the origin.

Proof. It suffices, according to Theorem 2, to show that $F_{q}(x y)$ is ETP on $\boldsymbol{R}$. This is shown by induction on $q$. For $q=0$ the theorem trivially holds true for $F_{0}(x y)={ }_{0} F_{0}(-;-; x y)=e^{x y}$. Assume now that $F_{q}(x y)$ is ETP on $\boldsymbol{R}$. According to (14.3) for $\alpha>0$

$$
\begin{aligned}
& { }_{q+1} F_{q+1}\left[\begin{array}{l}
\alpha, \quad \alpha_{1}, \cdots, \alpha_{q} \\
\alpha+1, \alpha_{1}+1, \cdots, \alpha_{q}+1 ;
\end{array} x y\right]=\frac{1}{B(\alpha, 1)} \int_{0}^{1} t^{\alpha-1} F_{q}(x y t) d t \\
& =\frac{x^{-\alpha}}{\alpha} \int_{0}^{x} F_{q}(y t) t^{\alpha-1} d t \\
& =\int_{0}^{\infty} F_{q}(y t) K(t, x) d \mu(t) \text {, }
\end{aligned}
$$

where $x>0, d \mu(t)=(1 / \alpha) t^{\alpha-1} d t, \alpha>0$ and

$$
K(t, x)= \begin{cases}x^{-\alpha}, & 0<t \leqq x \\ 0, & \infty>t>x>0 .\end{cases}
$$


$K(t, x)$ is TP on $(0, \infty) \times(0, \infty)\left[9\right.$, p. 102]. Since $F_{q}(y t)$ is ETP on $(-\infty, \infty) \times(0, \infty)$ it follows easily from the composition formula that ${ }_{q+1} F_{q+1}(x y)$ is $\mathrm{STP}$ on $(0, \infty) \times(-\infty, \infty)$. The analyticity of this kernel implies [9, p. 50] that it is in fact ETP on $(0, \infty) \times(-\infty, \infty)$. For $x<0$ we have

$$
{ }_{q+1} F_{q+1}(x y)=\int_{0}^{\infty} F_{q}(-y t) K(t,-x) d \mu(t) .
$$

Here $K(t,-x)$ is $R R$ on $(0, \infty) \times(-\infty, 0)$. Therefore, as before, ${ }_{q+1} F_{q+1}(x y)$ is ETP on $(-\infty, 0) \times(-\infty, \infty)$. By continuity ${ }_{q+1} F_{q+1}(x y)$ is ETP on $(-\infty, \infty) \times(-\infty, \infty)$ as required.

A more general theorem holds for the case $p=q+1$, however the proof is virtually the same.

THEOREM 7. The following hold

(i ) Let $F_{q}(z \bar{\zeta})={ }_{q+1} F_{q}\left[\begin{array}{l}\alpha_{1}, \cdots, \alpha_{q}, \alpha_{0} ; z \bar{\zeta} \\ \alpha_{1}+1, \cdots, \alpha_{q}+1 ; \alpha_{j}>0, j=1, \cdots, q\end{array}\right.$ and $\alpha_{0}$ any real number $\neq 0,-1,-2, \cdots$. Then $F_{q}(z \bar{\zeta})$ is ESR on 4. For $\alpha_{0}>0, F_{q}(z \bar{\zeta})$ is the reproducing kernel of the Hilbert space of analytic functions in $\triangle$ normed by (14.2) and is ETP on all diameters of 4 . For $\alpha_{0}<0$ ( $\alpha_{0}$ not an integer) $F_{q}(z \bar{\zeta})$ is ESR on the above diameters with the mth order signature

$$
\varepsilon_{m}\left(F_{q}\right)=(-1)^{\left(\left[-\alpha_{0}\right]+1\right)\left(m-1-\left[-\alpha_{0}\right] / 2\right)} .
$$

(ii) Let $G_{q}(z \bar{\zeta})={ }_{q+1} F_{q}\left[\begin{array}{l}\alpha_{1} \\ \left.\alpha_{1}+1, \cdots, \alpha_{q-1}, \quad 2 \alpha_{0}, \quad \alpha_{0} ; z \bar{\zeta}\right], \alpha_{q-1}+1, \alpha_{0}+1 ;\end{array}\right.$ $j=1, \cdots, q-1$ and $\alpha_{0} \neq 0,-1,-2, \cdots$. Then $G_{q}(z \bar{\zeta})$ is ESR on $\Delta$ and ETP on all diameters of $\Delta$. Moreover, for $0 \neq \alpha_{0}>-1 / 2, G_{q}(z \bar{\zeta})$ is the producing kernel of the Hilbert space of analytic functions in $\triangle$ normed by (14.2).

(iii) Let $H_{q}(z \bar{\zeta})={ }_{q+1} F_{q}\left[\begin{array}{l}\alpha_{1} \\ \alpha_{1}+1, \cdots, \alpha_{q-1}, 2 \alpha_{0}, \alpha_{0}+1 ; z \bar{\zeta}\end{array}\right], \alpha>0, j=1$, $\cdots, q-1$ and $\alpha_{0} \neq-n / 2, n=1,2, \cdots$. Then $H_{q}(z \bar{\zeta})$ is ESR on $\Delta$ and ETP on all diameters of $\Delta$. Moreover, for $\alpha_{0}>-1 / 2, H_{q}(z \bar{\zeta})$ is the reproducing kernel of the Hilbert space of analytic functions in $\triangle$ normed by (14.2).

Proof. Again it suffices to prove that the kernels in question are ESR on the real diameter of $\Delta,(-1,1)$. The proof is carried by induction on $q$.

(i) For $q=0, F_{0}(x y)={ }_{1} F_{0}\left(\alpha_{0} ;-; x y\right)=(1-x y)^{-\alpha_{0}}$ and so by Corollary 5 (or Theorem 5 ) the statement holds true for $q=0$. Assume the statement is true for $q$. According to (14.3) for $\alpha>0$ 


$$
\begin{aligned}
{ }_{q+2} F_{q+1}(x y) & ={ }_{q+2} F_{q+1}\left[\begin{array}{l}
\alpha_{1}, \\
\alpha_{1}+1, \cdots, \alpha_{q}, \quad \alpha ; \alpha_{q}+1, \alpha+1 ;
\end{array}\right] \\
& =\frac{1}{B(\alpha, 1)} \int_{0}^{1} t^{\alpha-1} F_{q}(x y t) d t \\
& =\frac{x^{-\alpha}}{\alpha} \int_{0}^{x} F_{q}(y t) t^{\alpha-1} d t \\
& =\int_{0}^{1} F_{q}(y t) K(t, x) d \mu(t),
\end{aligned}
$$

where $x>0, d \mu(t)=(1 / \alpha) t^{\alpha-1} d t, \alpha>0$ and

$$
K(t, x)= \begin{cases}x^{-\alpha}, & 0<t \leqq x \\ 0, & 1>t>x>0 .\end{cases}
$$

$K(t, x)$ is $\mathrm{TP}$ on $(0,1) \times(0,1)$. Assume first that $\alpha_{0}>0$. Since $F_{q}(y t)$ is ETP on $(-1,1) \times(0,1)$ it follows from the composition formula that ${ }_{q_{+2}} F_{q_{+1}}(x y)$ is $\operatorname{STP}$ on $(0,1) \times(-1,1)$. The analyticity of ${ }_{q+2} F_{q+1}(x y)$ implies that it is in fact $\operatorname{ETP}$ on $(0,1) \times(-1,1)$. For $x<0$ we have

$$
{ }_{q+2} F_{q+1}(x y)=\int_{0}^{1} F_{q}(-y t) K(t,-x) d \mu(t) .
$$

But $K(t,-x)$ is $\mathrm{RR}$ on $(0,1) \times(-1,0)$ and $F_{q}(-y t)$ is ERR on $(-1,1) \times(0,1)$. Hence as before ${ }_{q+2} F_{q+1}(x y)$ is $\operatorname{ETP}$ on $(-1,0) \times(1,1)$. By continuity ${ }_{q+2} F_{q+1}(x y)$ is $\operatorname{ETP}$ on $(-1,1) \times(-1,1)$ as required. The same applies when $\alpha_{0}<0$ ( $\alpha_{0}$ not an integer).

(ii) The case $q=1$ holds true, for then,

$$
G_{1}(z \bar{\zeta})={ }_{2} F_{1}\left(2 \alpha_{0}, \alpha_{0}^{\prime} ; \alpha_{0}+1 ; z \bar{\zeta}\right)
$$

and the statement was verified in (13.1), (13.2) and Corollary 7. The induction now carries exactly as in (i).

(iii) The case $q=1$ was shown in (13.5) and (13.6). Indeed, in this case $H_{1}(z \bar{\zeta})={ }_{2} F_{1}\left(2 \alpha_{0}, \alpha_{0}+1 ; \alpha_{0} ; z \bar{\zeta}\right)=(1+z \bar{\zeta})(1-z \bar{\zeta})^{-\left(2 \alpha_{0}+1\right)}$ and again the induction carries as before. This completes the proof of the theorem.

\section{REFERENCES}

1. M. Abramowitz and I. A. Stegun, Handbook of Mathematical Functions, Appl. Math. Ser. 55, Natl. B. of Standards, Washington, 1964.

2. R. Askey and S. Wainger, A convolution structure for Jacobi series, Amer. J. Math., 91 (1969), 463-485.

3. H. Bateman, A generalization of the Legendre polynomial, Proc. London Math. Soc., (2), 3 (1905), 111-123.

4. S. Bergman, The Kernel Function and Conformal Mapping, Math. Surveys 5, Amer. Math. Soc., Providence, 1970. 
5. J. Burbea, Total positivity and reproducing kernels, Pacific J. Math., 55 (1974), 343-359.

6. A. Erdely, Higher Transcendental Functions I, McGraw-Hill, New York, 1953.

7. - Higher Transcendental Functions II, McGraw-Hill, New York, 1953.

8. R. Horton, Jacobi polynomials, IV; a family of variation diminishing kernels, Siam J. Math. Anal., 6 (1975), 544-550.

9. S. Karlin, Total Positivity, Vol. 1, Stanford Univ. Press, Stanford, 1968.

10. - Best quadrature formulas and splines, J. Approximation Theory, 4 (1971), 59-90.

11. R. E. A. C. Paley and N. Wiener, Fourier Transforms in the Complex Domain, Colloquium Publications, Vol. 19, Amer. Math. Soc., New York, 1934.

12. G. Pólya and I. J. Schoenberg, Remarks on the la Vallée Poussin means and convex conformal maps of the circle, Pacific J. Math., 8 (1958), 295-334.

13. E. D. Rainville, Special Functions, Macmillan, New York, 1960.

14. A. Selberg, Automorphic functions and integral operators, Seminars on analytic functions, Institute for Advanced Study, Princeton, 1957, Vol. II, 152-161.

15. H. S. Shapiro and A. L. Shields, On the zeros of functions with finite Dirichlet integral and some related function spaces, Math. Z., 80 (1962), 217-229.

Received December 4, 1975. This work is supported by the U. S. Army Grant No. 2065.

UNiversity of Pittsburgh 


\section{PACIFIC JOURNAL OF MATHEMATICS}

EDITORS

RICHARD ARENS (Managing Editor)

University of California

Los Angeles, California 90024

R. A. BEAUMONT

University of Washington

Seattle, Washington 98105

J. DugundjI

Department of Mathematics

University of Southern California

Los Angeles, California 90007

D. Gilbarg and J. Milgram

Stanford University

Stanford, California 94305

\section{ASSOCIATE EDITORS}

E. F. BeCKENBACH

B. H. NeUMaNN

F. WOLF

K. YoSHIDA

\section{SUPPORTING INSTITUTIONS}

UNIVERSITY OF BRITISH COLUMBIA

CALIFORNIA INSTITUTE OF TECHNOLOGY

UNIVERSITY OF CALIFORNIA

MONTANA STATE UNIVERSITY

UNIVERSITY OF NEVADA

NEW MEXICO STATE UNIVERSITY

OREGON STATE UNIVERSITY

UNIVERSITY OF OREGON

OSAKA UNIVERSITY

\author{
UNIVERSITY OF SOUTHERN CALIFORNIA \\ STANFORD UNIVERSITY \\ UNIVERSITY OF TOKYO \\ UNIVERSITY OF UTAH \\ WASHINGTON STATE UNIVERSITY \\ UNIVERSITY OF WASHINGTON \\ AMERICAN MATHEMATICAL SOCIETY \\ NAVAL WEAPONS CENTER
}




\section{Pacific Journal of Mathematics \\ Vol. 67, No. $1 \quad$ January, 1976}

Gregory Wayne Brumfiel and John W. Morgan, Homotopy theoretic

consequences of $N$. Levitt's obstruction theory to transversality for

spherical fibrations . ................................ 1

Jacob Burbea, Total positivity of certain reproducing kernels ........... 101

Wai-Mee Ching, The structure of standard $C^{*}$-algebras and their

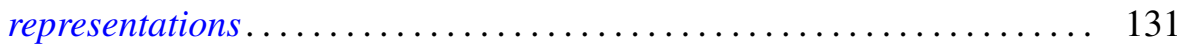

Satya Deo, The cohomological dimension of an $n$-manifold is $n+1 \ldots \ldots 155$

Masahiko Fujiwara and Masaki Sudo, Some forms of odd degree for which the Hasse principle fails ................................. 161

Mikihiro Hayashi, Smoothness of analytic functions at boundary points ... 171

Rebecca A. Herb, A uniqueness theorem for tempered invariant

eigendistributions ................................ 203

David Alan Legg, Orlicz space convergence of martingales of

Radon-Nikodým derivatives given a $\sigma$-lattice.................. 209

D. B. McAlister, v-prehomomorphisms on inverse semigroups......... 215

Bruno J. Mueller, Localization in fully bounded Noetherian rings . ........ 233

Donald J. Newman and A. R. Reddy, Rational approximation to $x^{n} \ldots \ldots .247$

Abraham Ziv, Inclusion relations between power methods of limitation.... 251 